\title{
PROTOPLANETARY AND TRANSITIONAL DISKS IN THE OPEN STELLAR CLUSTER IC 2395
}

\author{
Zoltan Balog $^{1}$, Nick Siegler ${ }^{2}$, G. H. Rieke ${ }^{3}$, L. L. Kiss ${ }^{4}$, James Muzerolle $^{5}$, R. A. Gutermuth ${ }^{6}$, Cameron P. M. Bell ${ }^{7}$, \\ J. VINKÓ ${ }^{8}$, K. Y. L. $\mathrm{SU}^{3}$, E. T. YOUNG ${ }^{9}$, AND ANDRÁs GÁsPÁR ${ }^{3}$ \\ ${ }^{1}$ Max Planck Institute for Astronomy, Heidelberg, D-69117, Germany; balog@ mpia-hd.mpg.de \\ ${ }^{2}$ NASA Exoplanet Exploration Program, Jet Propulsion Laboratory, California Institute of Technology, 4800 Oak Grove Drive, Pasadena, CA 91109, USA \\ ${ }^{3}$ Steward Observatory, 933 N. Cherry Ave, University of Arizona, Tucson, AZ 85721, USA \\ ${ }^{4}$ Konkoly Observatory, Research Center for Astronomy and Earth Sciences, P.O. Box 67, H-1525 Budapest, Hungary \\ ${ }^{5}$ Space Telescope Science Institute, 3700 San Martin Drive, Baltimore, MD 21218, USA \\ ${ }^{6}$ Department of Astronomy, University of Massachusetts, Amherst, MA, USA \\ ${ }^{7}$ Institute for Astronomy, ETH Zürich, Wolfgang-Pauli-Strasse 27, 8093, Zürich, Switzerland \\ ${ }^{8}$ Dept. of Optics and Quantum Electronics, University of Szeged, H-6720 Szeged, Hungary \\ ${ }^{9}$ NASA Ames SOFIA Science Center, N211, Mountain View, CA 94043, USA \\ Received 2016 June 5; revised 2016 September 9; accepted 2016 September 9; published 2016 November 18
}

\begin{abstract}
We present new deep UBVRI images and high-resolution multi-object optical spectroscopy of the young ( $\sim 6-10 \mathrm{Myr}$ old), relatively nearby $(800 \mathrm{pc})$ open cluster IC 2395 . We identify nearly 300 cluster members and use the photometry to estimate their spectral types, which extend from early B to middle M. We also present an infrared imaging survey of the central region using the IRAC and MIPS instruments on board the Spitzer Space Telescope, covering the wavelength range from 3.6 to $24 \mu \mathrm{m}$. Our infrared observations allow us to detect dust in circumstellar disks originating over a typical range of radii from $\sim 0.1$ to $\sim 10$ au from the central star. We identify 18 Class II, 8 transitional disk, and 23 debris disk candidates, respectively, 6.5\%, 2.9\%, and $8.3 \%$ of the cluster members with appropriate data. We apply the same criteria for transitional disk identification to 19 other stellar clusters and associations spanning ages from $\sim 1$ to $\sim 18 \mathrm{Myr}$. We find that the number of disks in the transitional phase as a fraction of the total with strong $24 \mu \mathrm{m}$ excesses ([8] - [24] $\geqslant 1.5)$ increases from $(8.4 \pm 1.3) \%$ at $\sim 3 \mathrm{Myr}$ to $(46 \pm 5) \%$ at $\sim 10 \mathrm{Myr}$. Alternative definitions of transitional disks will yield different percentages but should show the same trend.
\end{abstract}

Key words: circumstellar matter - infrared: stars - open clusters and associations: individual (IC 2395) - stars: premain sequence

Supporting material: machine-readable tables

\section{INTRODUCTION}

The Spitzer Space Telescope (Spitzer; Werner et al. 2004) has significantly improved our understanding of how protoplanetary disks form, evolve, and eventually dissipate. By $15 \mathrm{Myr}$ the accretion of gas onto protostars has largely ceased and most primordial disks have dissipated (e.g., Haish et al. 2001; Mamajek et al. 2004; Meng et al. 2016). By this time, planetesimals have formed and dust produced in their collisions yields planetary debris disks. These regenerated disks indirectly reveal the presence of planetary bodies required to replenish the dust and allow us to trace the evolution of planetary systems over the full range of stellar ages (e.g., Lagrange et al. 2000; Dominik \& Decin 2003; Wyatt 2008; Gáspár et al. 2013; Sierchio et al. 2014).

The beginning of the transition from an optically thick accretion disk to an optically thin debris disk occurs from the inside out (e.g., Skrutskie et al. 1990; Megeath et al. 2005; Sicilia-Aguilar et al. 2005; Muzerolle et al. 2010; Espaillat et al. 2014) and is marked by a characteristic spectral energy distribution (SED) with little excess at shorter $(<6 \mu \mathrm{m})$ wavelengths but still retaining strong emission at the longer ones. These "transitional" disks appear to represent the process of clearing "caught in the act." The result is a largely evacuated inner region accompanied by an optically thick primordial disk at larger radii. This phase is crucial to our understanding of disk dissipation and planet formation because it signals the end of stellar accretion and the consumption of nearly all the gas in the disk. Given the small number of transitional disks identified relative to the number of primordial and debris disks, it has been concluded that this phase is of short duration, of the order of a few hundred thousand years (Skrutskie et al. 1990; Kenyon \& Hartmann 1995; Simon \& Prato 1995; Wolk \& Walter 1996; Muzerolle et al. 2010; Espaillat et al. 2014).

The key time period to observe the transitional phase is from a few to about $15 \mathrm{Myr}$. Clusters and associations are ideal laboratories for studying disk evolution because the member stars are coeval to within a few million years, of similar composition and reddening, at similar and reliably measured distance, and numerous enough to have a wide range of masses and to support the drawing of statistically valid conclusions. Unfortunately, there are only a few appropriately aged young clusters within a kiloparsec to support characterization of the transitional disk phase. For more distant clusters, Spitzer has insufficient sensitivity in the mid-infrared to measure the photospheres of the lowest-mass members and to identify complete samples of transitional disks.

The open cluster IC 2395 can augment studies of this phase of disk evolution. The cluster is $800 \mathrm{pc}$ distant (Section 3.3, Claria et al. 2003). Despite its proximity, it has not been extensively studied. Claria et al. (2003) conducted the largest photometric investigation of the cluster, identifying candidate members and estimating the cluster's age, distance, extinction, and angular size. Sensitive to a limiting magnitude of $V<15 \mathrm{mag}$, their survey found 78 probable and possible members through $U B V$ photometry. There have also been several proper-motion studies but none combined with 
photometric data. The cluster age has been estmated at $6 \pm 2$ Myr (Claria et al. 2003) on the traditional calibration for ages of young clusters and associations (e.g., Mamajek 2009). A revised age calibration has been proposed (e.g., Pecaut et al. 2012; Bell et al. 2013, 2015), on which we derive in this paper an age of $\sim 9 \mathrm{Myr}$ (Section 3.3). On either calibration, IC 2395 is in the critical range to characterize transitional disk behavior.

To increase our understanding of this cluster, we have obtained fields $\sim 45$ arcmin square of deep optical (UBVRI) and mid-IR $(3.6,4.5,5.8,8.0$, and $24 \mu \mathrm{m})$ photometry and highresolution optical spectroscopy of IC 2395. We describe the new observations in Section 2 and discuss the cluster membership and age in Section 3. In Section 4, we identify and characterize the circumstellar disks in the cluster with emphasis on identifying transitional disks. We combine these results with a homogeneous treatment of transitional disks in 19 other young clusters and associations in Section 5, to probe the evolution of circumstellar disks through the transitional phase. We summarize and conclude the paper in Section 6.

\section{OBSERVATIONS AND SAMPLE SELECTION}

In this section, we discuss the Spitzer observations and data reduction for IC 2395, as well as our optical UBVRI observations and spectroscopy of selected probable members. In addition to the photometry described below, we took $J H K$ measurements from 2MASS. We also re-examine the results of Claria et al. (2003) and include appropriate members from their work in our study.

\subsection{Spitzer/IRAC}

IC 2395 was observed using IRAC (Fazio et al. 2004) on Spitzer in 2003 December as part of a GTO program (PID 58, PI Rieke, Evolution and Lifetimes of Protoplanetary Disks) to study protoplanetary disks and dust evolution. The survey covers an area of $\approx 44^{\prime} \times 44^{\prime}(9 \times 9$ grid, $\sim 0.54$ square degrees) in each of the four IRAC channels. The $12 \mathrm{~s}$ highdynamic-range mode was used to obtain two frames in each position, one with an exposure time of $0.4 \mathrm{~s}$ and one with 10.4 s. The observation of each field was repeated with a small offset, providing an integration time of $20.8 \mathrm{~s}$ for each position. The frames were processed using the Spitzer Science Center IRAC Pipeline v14.0, and mosaics were created from the basic calibrated data frames using a custom IDL package, Cluster Grinder, that treats bright source artifacts and removes cosmicray hits and spatial scale distortion during mosaic construction (Gutermuth et al. 2009). Due to the 7 arcmin offset between channels $1 / 3$ and channels $2 / 4$, the total area covered in all four channels is about 0.37 square degrees.

The IDL-based photometry visualization tool PhotVis version 1.10 (Gutermuth et al. 2008) was used on the reduced images to find sources and carry out aperture photometry on them. The radii of the source aperture and of the inner and outer boundaries of the sky annulus were $2.4,2.4$, and 7.2 arcsec, respectively. The calibration was based on large-aperture measurements of standard stars. The zero-point magnitudes of the calibration were 19.6642, 18.9276, 16.8468, and 17.3909, corresponding to zero-point fluxes of 280.9, 179.7, 115.0, and 64.13 Jy for channels 1, 2, 3, and 4, respectively (Reach et al. 2005). Corrections of $0.21,0.23,0.35$, and 0.5 mag were applied for channels $1,2,3$, and 4, respectively, to correct for the differences between the aperture sizes used for the IC 2395 sources and for the standard stars.

\subsection{Spitzer/MIPS}

IC 2395 was also observed using MIPS on Spitzer in 2003 as part of the same GTO program. MIPS is equipped with a threechannel camera with central wavelengths of approximately 24 , 70, and $160 \mu \mathrm{m}$ (Rieke et al. 2004). The longer-wavelength channels are insensitive to stellar photospheric emission at the distance of IC 2395 and no cluster stars were detected at $70 \mu \mathrm{m}$ or $160 \mu \mathrm{m}$. This study is based on only the MIPS $24 \mu \mathrm{m}$ channel.

The observations used the medium scan mode with halfarray cross-scan offsets, resulting in a total exposure time per pixel of $80 \mathrm{~s}$. The images were processed using the Data Analysis Tool of the MIPS instrument team (Gordon et al. 2005), which calibrates the data, corrects distortions, and rejects cosmic rays during the coadding and mosaicking of individual frames. A column-dependent median subtraction routine was applied to remove any residual patterns from the individual images before combining them into the final $24 \mu \mathrm{m}$ mosaic. The total area mapped was nearly a square degree $\left(89^{\prime} \times 40^{\prime}\right)$.

We measured the $24 \mu \mathrm{m}$ flux density of individual sources using the standard photometry routine allstar in the IRAF data reduction package daophot, and within a $15^{\prime \prime}$ aperture. We then applied an aperture correction of 1.73 to account for the flux density outside the aperture, as determined from the STinyTim $24 \mu \mathrm{m}$ point-spread function (PSF) model (Engelbracht et al. 2007). Finally, fluxes were converted into magnitudes referenced to the Vega spectrum (with the zero point at $7.17 \mathrm{Jy}$ ). Typical $1 \sigma$ measurement uncertainties for the MIPS $24 \mu \mathrm{m}$ fluxes are $50 \mu \mathrm{Jy}$; there is also a $2 \%$ uncertainty in the absolute calibration (Engelbracht et al. 2007). The MIPS image is sufficiently sensitive to detect the photospheres of $\sim \mathrm{A} 0$ stars at the distance of IC 2395.

Figure 1 is a three-color composite image composed of IRAC wavelengths $3.6 \mu \mathrm{m}$ (blue) and $8 \mu \mathrm{m}$ (green) and MIPS $24 \mu \mathrm{m}$ (red). The shortest-wavelength channel reaches the photospheres of all stars in the cluster. In addition, however, it also picks up many background stars. The $8 \mu \mathrm{m}$ image shows fewer sources and highlights dust (and associated gas). This image likely has a contribution from polycyclic aromatic hydrocarbons and potentially also silicate emission at its longwavelength limit. At $24 \mu \mathrm{m}$, we see cooler extended dust and the cluster members with significant excess emission. The brightest source is EP Velorum, an M6, thermally pulsating star on the asymptotic giant branch (Kerschbaum \& Hron 1994), unassociated with the cluster. The $24 \mu \mathrm{m}$ mosaic of the central region of IC 2395 is displayed in Figure 2, with the most prominent infrared-excess sources marked.

\subsection{UBVRI Photometry}

Our optical observations were made with the SITe 2048-\#6 CCD camera on the $1.5 \mathrm{~m}$ telescope at Cerro Tololo InterAmerican Observatory (CTIO) on 2003 January 23 and 24 as part of a three-day campaign (2003 January 22, 23, and 24) to provide optical data in support of Spitzer observations for IC 2395 and NGC 2451 (discussed in a separate paper; Balog et al. 2009). The camera was mounted at the $f / 13.5$ focal position, covering a field of view of $15 \times 15 \operatorname{arcmin}^{2}$ with a 


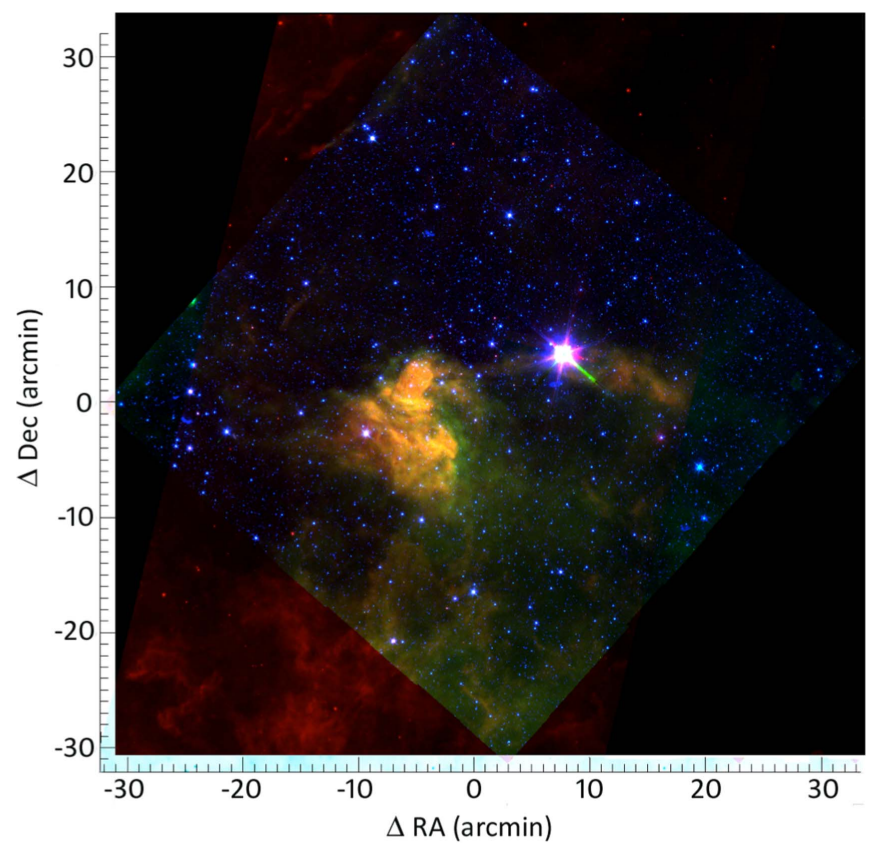

Figure 1. Three-color composite image of IC 2395 composed of IRAC wavelengths $3.6 \mu \mathrm{m}$ (blue) and $8.0 \mu \mathrm{m}$ (green) and MIPS $24 \mu \mathrm{m}$ (red). The coordinates are relative to R.A. $=130.6111$, decl. $=-48.1690$. Likely contributors to the emission in the $8.0 \mu \mathrm{m}$ channel are silicate grains and aromatic molecules, seen as the bright green areas.

resolution of $0.43 \mathrm{arcsec} / \mathrm{pixel}$ for the entire area of $2048 \times 2048$ pixel $^{2}$. The observations were made through Johnson-Cousins UBVRI filters, applying the Tek \#1 filter set. $^{10}$

The whole cluster was covered by $3 \times 3=9$ CCD frames centered on and around the brightest inner area at $\mathrm{R}$. A. $=08: 42: 30$, decl. $=-48: 06: 00$. One off-cluster area (separated by $\sim 1.5$ from the cluster center) was also imaged to sample the foreground/background object population in the same line of sight. Each field was imaged three times through the same filter. One frame was obtained with a short exposure time ( $10 \mathrm{~s}$ for $U$ and $5 \mathrm{~s}$ for $B V R I$ ) and the other two frames were taken with longer ones $(250 \mathrm{~s}$ for $U, 70 \mathrm{~s}$ for $B$, and $50 \mathrm{~s}$ for $V R I)$.

The reduction of the raw frames was performed with standard routines using IRAF. ${ }^{11}$ After trimming the edges of the frames and subtracting the bias level from each image, the frames were divided by a master flat-field image obtained by median-combining the available flat-field frames for each filter. Both dome flats and sky flats were taken at the beginning of each night and combined together into the master flat frames. After flat-field division, the two long-exposure frames corresponding to the same filter were averaged to increase the signalto-noise ratio.

The photometry of the cluster frames was conducted via PSF-fitting using DAOPHOT implemented in IRAF. A secondorder spatially variable PSF (varorder $=2$ ) was built for each frame to help compensate for the distortions of the PSFs due to either the optical imaging artifacts in the large field of view or

\footnotetext{
${ }^{10}$ http://www.ctio.noao.edu/noao/content/CTIO-3 $\times 3$-inch-and-4 $\times 4$-inchFilters

11 IRAF is distributed by NOAO, which is operated by the Association of Universities for Research in Astronomy (AURA) Inc. under cooperative agreement with the National Science Foundation.
}

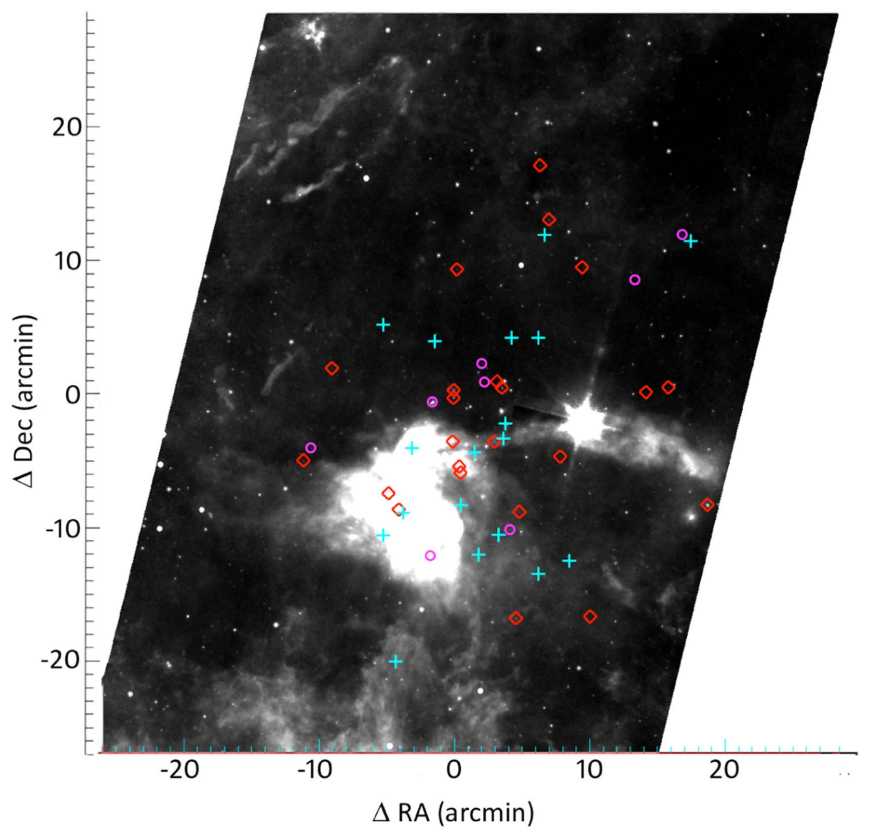

Figure 2. The central $\sim 60^{\prime} \times 40^{\prime}$ mosaic of IC 2395 taken with the MIPS $24 \mu \mathrm{m}$ channel. The coordinates are relative to R.A. $=130.6389$, decl. $=-48.0688$. Sources with disks are identified with symbols: cyan crosses represent Class II candidates, magenta circles represent transitional disk candidates, and red diamonds represent debris disk candidates. The point source FWHM is $5 ! 7$ and the plate scale is 1 ." $25 /$ pixel.

guiding errors that occured randomly on a few frames. The model function=penny 2 was selected to account for the slight elongation of the PSF. The PSF stars were selected interactively from a sample of the $\sim 100$ brightest, nonsaturated, well isolated stars on each frame, omitting the ones with suspicious profiles and/or detectable neighbors within $r=15$ pixels. The fitrad parameter was set according to the value of the FWHM. The detection threshold was fixed at the $4 \sigma$ level on each frame.

The transformation of the CTIO instrumental magnitudes into the standard Johnson-Cousins system was performed via the observations of Landolt photometric standard sequences (Landolt 1992). The description of the standard transformation is discussed in Balog et al. (2009).

We applied aperture corrections for each frame to match the PSF photometry to the aperture photometry obtained for standard stars. The aperture photometry was computed with $r_{\mathrm{ap}}=8$ pixels radius. The local sky level was estimated as the mode of the pixel distribution within an annulus having inner and outer radii of 10 and 20 pixels, respectively, centered on each object. Inspecting the final instrumental magnitudes, we found a very small $0.02-0.03$ mag systematic offset between the long- and short-exposure frames and also found that there is a $~ 0.03-0.1 \mathrm{mag}$ offset between the different frames of the mosaics. We tied our photometry to the middle frame (which overlaps with all of the remaining fields) of the $3 \times 3$ mosaic to ensure the consistency of our data set.

We tested the quality and stability of the photometry, including the standard transformation, by comparing our standard magnitudes with those from Claria et al. (2003) (unfortunately, only the $V$ and $B-V$ data could be compared in this way). We discovered systematic offsets of $\simeq 0.19$ mag and $\simeq 0.015 \mathrm{mag}$ between the two data sets in $V$ and $B-V$, respectively. Claria et al. (2003) report that there were 
systematic differences (sometimes as large as $0.2 \mathrm{mag}$ ) between their photometry and earlier work. We therefore also compared our photometry with stars from the field found in the SIMBAD database. The systematic offsets were smaller, but the scatter of the data was much larger due to the non-uniformity of the SIMBAD data. However, we used the exact same calibration in the case of NGC 2451 (Balog et al. 2009), where we found an almost perfect agreement with the previously published data set of Platais et al. (2001). In cases where an object is missing from our photometric sample we adjust its photometry from Claria et al. (2003) to match ours and give that value in the summary table for all the members.

\subsection{Spectroscopy}

We acquired AAOmega spectra using the Anglo-Australian Telescope at Siding Spring, Australia on three nights (2009 December 17-23) in conditions of clear skies with 1.5-2.5 arcsec seeing.

\subsubsection{Target Selection}

To make optimal use of the telescope time, we pre-selected member candidates based on their positions on the colormagnitude (CM) diagram and color-color (CC) diagram. The 2MASS near-infrared photometry in particular is useful in deselecting reddened background stars. We matched our optical photometry to the 2MASS positions and used the $V$ versus $V-K \mathrm{CM}$ diagram and the $V-K$ versus $V-I$ CC diagram to separate possible cluster members from the foreground and background population. We selected stars as member candidates if their positions on the CM diagram were compatible with the cluster age, distance, and reddening, allowing for errors due to binarity and age spread. First we combined the pre-main-sequence isochrones with ages 3 and 10 Myr (traditional age calibration) of Palla \& Stahler (1999) with the post-main-sequence isochrones of Marigo et al. (2008). We selected these isochrones because they bracket the age of the cluster ( $\sim 6 \mathrm{Myr}$ on this calibration) and allow some room for errors in the age estimates. Then we shifted these isochrones to the distance modulus of IC 2395 (800 pc) and applied additional shifts to take into account the reddening and extinction. We show the $\mathrm{CC}$ diagram with the selected possible members in Figure 3. We also included all objects that showed some level of IR excess in the IRAC bands even when they were not covered by our optical imaging. ${ }^{12}$ Altogether 710 candidates were selected based on the above criteria. We were able to obtain spectra of 675 of the candidates.

\subsubsection{Observations and Data Processing}

In the blue arm of the spectrograph, we used the $2500 \mathrm{~V}$ grating, providing $\lambda / \Delta \lambda=8000$ spectra between 4800 and $5150 \mathrm{~A}$. In the red arm we used the $1700 \mathrm{D}$ grating that has been optimized for recording the Ca II IR triplet region. The red spectra range from 8350 to $8790 \AA$, with $\lambda / \Delta \lambda=10,000$. This setup has the highest spectral resolution available with AAOmega, suitable for measuring stellar radial velocities. In

\footnotetext{
12 The objects without $V$ and $I$ photometry (numbers $1-4,111,134,167$, and 278 ) include one identified as a transitional disk (134), two as class II sources $(111,167)$, and one as a debris disk (278): the ratio of the number of transitional to class II sources is identical to the overall value, so the selection of these objects does not bias our results on the incidence of transitional systems.
}

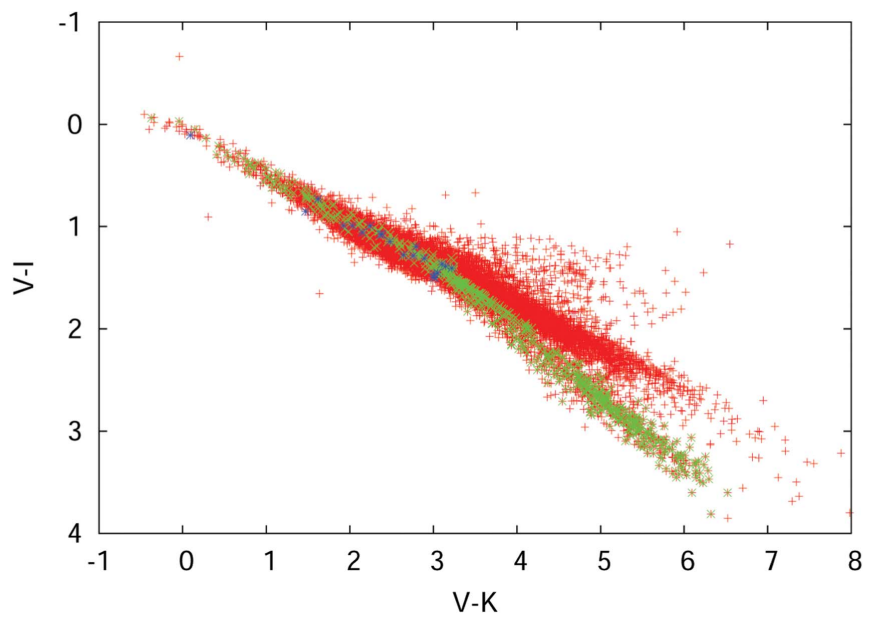

Figure 3. Optical-near-infrared CC diagram of member candidates of the IC 2395 cluster (green symbols) selected for the spectroscopic survey together with the complete sample of the photometric data (red symbols). Blue symbols show the stars with IR excess that may not be cluster members but were included in the spectroscopic sample.

total, we acquired 11 field configurations centered on the open cluster. The spectra were reduced using the standard TwoDegree Field data reduction pipeline. We performed continuum normalization for the stellar spectra using the IRAF task onedspec.continuum and then cleaned the strongest skyline residuals using linear interpolation of the surrounding continuum (see Balog et al. 2009 for a detailed description of the data processing of AAOmega).

Balog et al. (2009) also describe the methodology for determination of radial velocity. In summary, an iterative process was used to fit the atmospheric absorptions and the stellar radial velocity, based on synthetic stellar spectra. Our method is similar to that of the Radial Velocity Experiment project (Steinmetz et al. 2006; Zwitter et al. 2008), including use of the same library of synthetic spectra. We required three iterations to converge to a stable set of temperatures, surface gravities, metallicities, and radial velocities. We estimate the velocities to be accurate to within $\pm 1-2 \mathrm{~km} \mathrm{~s}^{-1}$ for the cooler stars $(T<8000-9000 \mathrm{~K})$ and $\pm 5 \mathrm{~km} \mathrm{~s}^{-1}$ for the hotter ones.

\section{CLUSTER MEMBERSHIP}

\subsection{High-mass Members from Claria et al. (2003)}

We now describe our identification of cluster members. Associating stars to stellar clusters can be challenging and is best carried out using several criteria, all of which need to be consistent with membership. The most confident membership designations are those that have photometric, kinematic, and spectroscopic measurements. In the case of IC 2395, no previous study has constructed a membership list based on multiple criteria.

Claria et al. (2003) conducted a $U B V$ investigation of the cluster's central $50^{\prime} \times 50^{\prime}$ region to a $V$-band limiting magnitude of 15 . This survey is the starting point for our identification of the high-mass cluster members. They selected cluster members photometrically by examining the positions of the observed stars in $U B V$ color-magnitude and color-color diagrams with respect to the theoretical models of Lejeune \& Schaerer (2001). Stars lying no more than $0.75 \mathrm{mag}$ above the zero-age main sequence (ZAMS) and deviating no more than 
Table 1

Studies of the Proper Motion of IC 2395

\begin{tabular}{|c|c|c|c|c|c|c|}
\hline Survey & $\begin{array}{c}\mu_{\alpha} \cos \delta \\
\left(\mathrm{mas}_{\mathrm{yr}} \mathrm{yr}^{-1}\right)\end{array}$ & $\begin{array}{l}\text { Uncertainty } \\
\left(\text { mas yr }^{-1}\right)\end{array}$ & $\begin{array}{c}\mu_{\delta} \\
\left(\operatorname{mas}_{\mathrm{yr}}^{-1}\right)\end{array}$ & 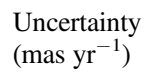 & $\begin{array}{l}\text { Sample } \\
\text { Size }\end{array}$ & Comments \\
\hline Gulyaev \& Nesterov (1992) & +2.3 & 1.0 & -1.8 & 1.0 & 8 & Greater than $3 \sigma$ away from this paper's result. \\
\hline Baumgardt et al. (2000) & -3.6 & 0.6 & 2.2 & 0.5 & 1 & $\ldots$ \\
\hline Dias et al. (2001) & -4.4 & 1.6 & 4.0 & 1.6 & 11 & $\ldots$ \\
\hline Dias et al. (2002) & -4.37 & 1.73 & 4.05 & 1.73 & 14 & $\cdots$ \\
\hline Loktin \& Beshenov (2003) & -4.86 & 0.33 & 3.48 & 0.23 & 33 & $\cdots$ \\
\hline Kharchenko et al. (2003) & -4.21 & 0.63 & 1.73 & 0.63 & 12 & $\ldots$ \\
\hline Kharchenko (2004) & -4.34 & 0.38 & 2.42 & 0.35 & 15 & $\cdots$ \\
\hline Dias et al. (2006) & -5.56 & 0.40 & 6.02 & 0.40 & 55 & Greater than $3 \sigma$ away from this paper's result. \\
\hline This paper & -3.92 & 0.33 & 3.03 & 0.26 & 11 & 1 clipped; values of proper motion used in this analysis. \\
\hline This paper & -3.60 & 0.36 & 2.69 & 0.28 & 10 & 2 clipped \\
\hline
\end{tabular}

0.10 mag from the locus of the $\mathrm{CC}$ main sequence were classified as cluster members. Claria et al. (2003) presented 61 sources meeting these photometric criteria. The positions in the $\mathrm{CM}$ and CC diagrams of another 16 stars were somewhat ambiguous but they were retained as possible members.

A comparison of 21 of these cluster members with a propermotion-selected list from Dias et al. (2001) showed very good agreement; however, the uncertainties in the mean propermotion survey are sufficiently large to make the comparison inconclusive. Without a kinematic, spectroscopic, or nearinfrared membership criterion to go along with the visible photometry, we believe that the classification of Claria et al. (2003) is insufficient for providing a robust list of bona fide cluster members.

\subsubsection{Mean Cluster Proper Motion}

We add a kinematic criterion to the photometric membership list of Claria et al. (2003) by selecting on proper motion. There are multiple estimates of the mean proper motion of IC 2395 members, but some of the estimates are inconsistent. We reestimated the mean proper motion using the spectroscopically confirmed sample of 14 B-type cluster members selected from Claria et al. (2003). The NOMAD catalog from USNO (Zacharias et al. 2004) provides the best available proper motion for each star (usually from Tycho-2 or Hipparcos; Hogg et al. 2000; Brown et al. 1997). Of the 14, 12 had proper motions, with the exceptions being HD 74455 and HD 74436. The variance-weighted mean $\mu_{\alpha} \cos \delta$ and $\mu_{\delta}$ values were calculated and the $\chi^{2}$ of each value was calculated for the sample. One star, HD 74251, was rejected because it contributed (by far) the majority of the $\chi^{2}$ for both $\mu_{\alpha} \cos \delta$ and $\mu_{\delta}$. Further clipping, however, had negligible effect on the final proper motion, so we calculated the mean value of proper motion for the remaining $11 \mathrm{~B}$-stars as representative for the group: $\left\langle\mu_{\alpha} \cos \delta\right\rangle=-3.9 \pm 0.4$ mas yr$^{-1}$ and $\left\langle\mu_{\delta}\right\rangle$ $=+3.0 \pm 0.4 \mathrm{mas} \mathrm{yr}^{-1}$. As the variance-weighted mean uncertainty $\left(\sim 0.3\right.$ mas $\left.\mathrm{yr}^{-1}\right)$ was close to the uncertainty in the proper motion of the Tycho-2 reference system $\left(\sim 0.25 \mathrm{mas} \mathrm{yr}^{-1}\right)$, we conservatively added that term in quadrature to derive our final estimate for the uncertainty $\left(\sim 0.4 \mathrm{mas} \mathrm{yr}^{-1}\right)$. The mean proper motion is consistent within the errors whether we calculate it as a true median (Gott et al. 2001), a Chauvenet-criterion clipped mean (Bevington \& Robinson 1992), or an unweighted mean, so our choice of $\mu$ estimation matters little.
Our derived mean proper motion agrees well with most of the previously measured values as shown in Table 1 (Baumgardt et al. 2000; Dias et al. 2001, 2002; Kharchenko et al. 2003, 2005; Loktin \& Beshenov 2003), but is severely at odds with the quoted values from Gulyaev \& Nesterov (1992) and Dias et al. (2006). The value of Dias et al. (2006) is dominated by large numbers of faint UCAC2 stars and likely suffers from a significant amount of contamination by field stars. As we (and most other studies) do not agree with the mean proper motion estimated by Dias et al. (2006), we do not use their membership probabilities.

\subsubsection{Revised High-mass Cluster Membership List}

We now take the 61 probable and 16 possible cluster members from Claria et al. (2003) and further select those as members that meet the combined near-infrared and optical photometric and the proper-motion criteria, i.e., those:

1. lying near a dereddened isochrone on near-IR and optical $\mathrm{CM}$ and $\mathrm{CC}$ diagrams,

2. with proper motions within $2 \sigma$ of our derived cluster mean, and

3. whose uncertainties of proper motion are less than 5 mas yr $^{-1}$.

All of the objects from Claria et al. (2003) were consistent with the photometric criterion. The second criterion was determined through a $\chi^{2}$ comparison with the mean cluster motion (as measured in Section 3.1.1 and presented in Table 1), which includes the objects' proper-motion uncertainty along with an assumed intrinsic velocity dispersion of $1 \mathrm{mas} \mathrm{yr}^{-1}$, where $1 \mathrm{mas} \mathrm{yr}^{-1} \approx 0.7 \mathrm{~km} \mathrm{~s}^{-1}$ (Bevington \& Robinson 1992). In equation form:

$$
\begin{aligned}
& \chi^{2}= \frac{\left[\overline{\left(\mu_{\alpha} \cos \delta\right)^{\mathrm{cl}}}-\left(\mu_{\alpha} \cos \delta\right)^{*}\right]^{2}}{\left(\sigma_{\mathrm{int}, \mu_{\alpha} \cos \delta}^{\mathrm{cl}}\right)^{2}+\left(\sigma_{\mu_{\alpha}}^{*} \cos \delta\right)^{2}} \\
&+\frac{\left[\overline{\mu_{\delta}^{\mathrm{cl}}}-\mu_{\delta}^{*}\right]^{2}}{\left(\sigma_{\mathrm{int}, \mu_{\delta}}^{\mathrm{cl}}\right)^{2}+\left(\sigma_{\mu_{\delta}}^{*}\right)^{2}},
\end{aligned}
$$

where the "cl" superscript designates the cluster, the "int" subscript designates intrinsic, and the asterisk superscript designates individual stars. By selecting those sources with $\chi^{2} \leqslant 6$ and two degrees of freedom, we expect only $\approx 5 \%$ of bona fide cluster members to be rejected using this criterion $(\sim 2 \sigma)$. 
We invoke the last criterion to reduce the chances that sources with relatively large uncertainties may unjustifiably obtain low $\chi^{2}$ values and contaminate our sample of bona fide

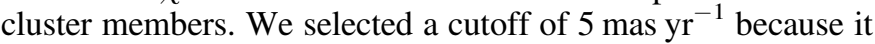
is less than the typical UCAC2 uncertainty for their faintest objects $(V \gtrsim 12)$. The consequence of this criterion, however, is that at the distance of IC 2395, spectral types inferred from $J-H$ to be roughly later than mid-F are deselected. In our effort to reduce interlopers, we have potentially removed faint cluster members, reducing both the sample size and mass range of a measured disk fraction. Incorporating later spectral types will require adding other criteria such as radial velocity, spectral classification, or spectral features pertaining to youth (see Section 3.2).

Our proper-motion criteria retained 40 from the sample of 61 probable cluster members of Claria et al. (2003). We examined the six sources that had $\chi^{2} \leqslant 6$ and uncertainties greater than $5 \mathrm{mas} \mathrm{yr}^{-1}$ and found that half of them had very inconsistent mean proper motions and were left deselected. The other three had mean proper motions within $1 \sigma$ of the cluster mean $\left(\chi^{2} \leqslant 1\right)$ and we reclassified them as possible cluster members. Another of the original 61 had no measured proper motion and was retained but only as a possible member. We also examined the sources that had $\chi^{2} \geqslant 6$ and uncertainties less than $5 \mathrm{mas} \mathrm{yr}^{-1}$ to identify any sources that were potentially penalized for having abnormally small reported uncertainties

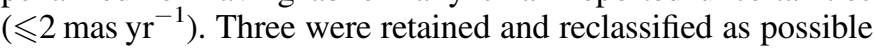
cluster members.

Of the original sample from Claria et al. (2003) of 16 possible cluster members, three were selected by proper motion and hence upgraded to probable members. One possible member had no measured proper motion and remains a possible member.

After having applied the additional membership criteria, we end up with 43 probable cluster members and 14 possible members. As we discuss below, we believe that two of the possible members are probable members, increasing the reported number to 45 cluster members and 12 possible members. In the following section, we discuss the selection of members from radial velocities measured from our spectra. In addition to allowing us to extend the membership list to lower masses, we apply this test to the possible massive members from Claria et al. (2003), eliminating a total of 10 probable and possible members. ${ }^{13}$

\subsection{Low-mass Member Candidates from Radial Velocities}

\subsubsection{Radial Velocities}

The result of our radial velocity survey is shown in Figure 4. The cluster members are clearly concentrated around $24.7 \mathrm{~km} \mathrm{~s}^{-1}$ with $\sigma=1.54 \mathrm{~km} \mathrm{~s}^{-1}$. We accepted an object as a cluster member if its radial velocity is within $2.4 \sigma(=1$ full width at half maximum of the distribution) of the mean radial velocity. ${ }^{14}$ Increasing this criterion to $3 \sigma$ admits 17 additional sources, but nine are likely to be non-members, where we have estimated the number of non-members as the average for all

\footnotetext{
${ }^{13}$ However, the selection criterion for radial velocity is biased against close massive binaries, so some of the eliminated stars may in fact be cluster members.

${ }^{14}$ We relaxed the radial velocity requirement if the object appeared to be a member or possible member based on proper-motion data. In this case we accepted a star as a member for the final analysis if its radial velocity was within $4.8 \sigma$ of the mean radial velocity.
}

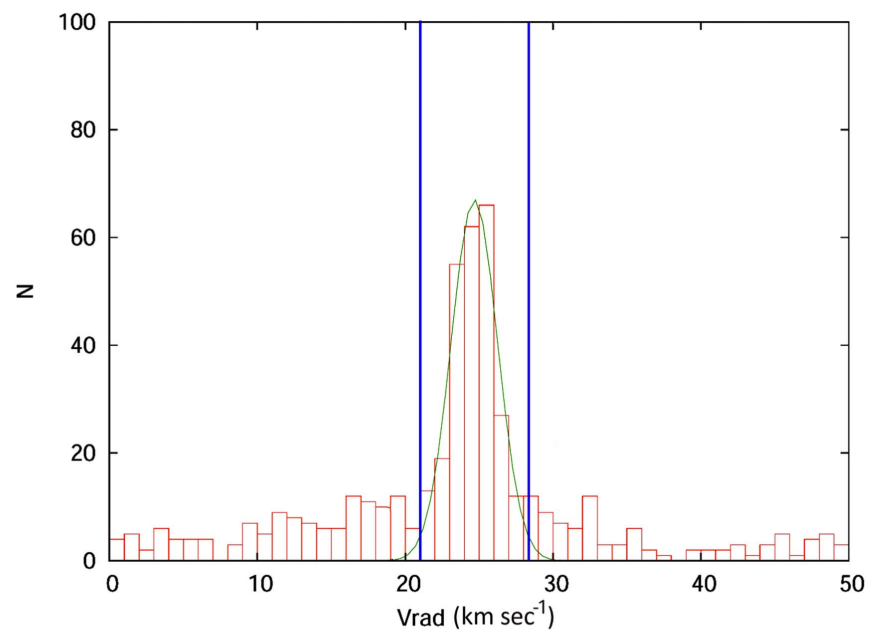

Figure 4. Radial velocity distributions toward IC 2395. The Gaussian fit is centered at $24.7 \mathrm{~km} \mathrm{~s}^{-1}$. The vertical blue lines indicate \pm one full width at half maximum above and below the central velocity. This velocity range was used to identify cluster members; it corresponds to $\pm 2.4 \sigma$.

velocities in the figure outside $4 \sigma$ from $24.7 \mathrm{~km} \mathrm{~s}^{-1}$. Increasing the window to $4 \sigma$ admits a total of 46 additional stars, but by the same method we estimate that 24 are likely to be nonmembers. Close binaries will have discrepant velocities outside our adopted criterion for cluster membership and will be rejected on this basis; it appears that there are relatively few such cases, or extending the selection threshold for radial velocity would add more probable members.

We also revised the high-mass membership of the propermotion members based on radial velocities, reducing the total size of this sample to 37 members and 10 possible members. The final selection of members from Claria et al. (2003) is listed in Table 2, while the full membership list including those from Table 2 is provided in Tables 3 and 4. Figure 6 places the members on $\mathrm{CM}$ and $\mathrm{CC}$ diagrams. Altogether we identified 250 low-mass members that were not included in the sample of proper motions, based on our radial velocity survey.

\subsubsection{Spectral Types}

Spectral types were assigned as available from the literature, as indicated in Table 2 . The $U-B$ versus $B-V$ color-color diagram shows that the reddening is uniform across the region, such that the $U-B$ versus $B-V$ sequence is well defined and there are no obvious signs of spread (outside of expectations from the presence of binaries) (Claria et al. 2003). We estimated the $E_{B-V}$ for the members with spectral types using the intrinsic main-sequence relation of Pecaut \& Mamajek (2013). Reddening this sequence according to $E_{U-B}=0.73 E_{B-V}$, our estimate is $E_{B-V}=0.09 \mathrm{mag}$, as also found by Claria et al. (2003). We therefore adopt an extinction equivalent to $E_{B-V}=0.09$.

Where types were unavailable, we used our photometry to estimate them. For the stars earlier than $\sim \mathrm{K} 4$ the preferred color for this purpose, $V-J$ or $V-K$, depends on the age of the cluster and whether there is, for example, active accretion. $V-K$ is the better choice for evolved field stars because of the longer-wavelength baseline, whereas for very young starforming clusters $V-J$ is preferred to circumvent excess emission at $K$. IC 2395 is in between. Therefore, before selecting the bands we did a number of tests. First, we 
Table 2

General Characteristics of IC 2395 Cluster Members from Claria et al. (2003)

\begin{tabular}{|c|c|c|c|c|c|c|c|c|c|c|}
\hline $\mathrm{ID}^{\mathrm{a}}$ & $\underset{\text { Table } 4^{\mathrm{b}}}{\text { ID }}$ & R.A. (2000) & Decl. (2000) & SpT & Ref. & $\begin{array}{c}V \\
(\mathrm{mag})\end{array}$ & $\begin{array}{l}B-V \\
(\mathrm{mag})\end{array}$ & $\begin{array}{c}\mu_{\alpha} \cos \delta \\
\left(\mathrm{mas} \mathrm{yr}^{-1}\right)\end{array}$ & $\begin{array}{c}\mu_{\delta} \\
\left(\operatorname{mas}_{\mathrm{yr}}^{-1}\right)\end{array}$ & $\begin{array}{l}\text { Other } \\
\text { Designation }^{c}\end{array}$ \\
\hline 246 & 1 & 8:40:09.9 & $-48: 07: 57.6$ & $\mathrm{~A} 5 \mathrm{~V}$ & 1 & 11.29 & 0.10 & $-3.4 \pm 2.9$ & $4.2 \pm 3.8$ & $\ldots$ \\
\hline 232 & 2 & 8:40:18.9 & $-48: 29: 43.4$ & A7V & 1 & 11.30 & 0.16 & $-8.1 \pm 1.4$ & $5.7 \pm 1.4$ & CD-484010 \\
\hline 229 & 3 & $8: 40: 25.6$ & $-48: 22: 48.1$ & F7 & 3 & 12.96 & 0.56 & $-13.7 \pm 5.8$ & $12.7 \pm 5.8$ & $\cdots$ \\
\hline 113 & 14 & $8: 40: 46.8$ & $-48: 12: 42.8$ & B8: & 2 & 9.60 & 0.05 & $-8.7 \pm 3.0$ & $-1.6 \pm 3.0$ & JW8, CD-474208 \\
\hline 112 & 16 & $8: 40: 48.2$ & $-48: 14: 15.6$ & A4 & 3 & 11.75 & 0.17 & $-6.1 \pm 2.3$ & $2.0 \pm 3.2$ & JW5, CD-474209 \\
\hline 111 & 23 & $8: 40: 53.4$ & $-48: 13: 31.8$ & B2IV & 2 & 6.96 & -0.16 & $-3.6 \pm 0.6$ & $2.2 \pm 0.4$ & JW2, HD 74234 \\
\hline 260 & $\cdots$ & $8: 40: 59.8$ & $-47: 47: 02.8$ & F0II & 1 & 11.39 & 0.17 & $-4.6 \pm 2.1$ & $3.9 \pm 2.0$ & CD-474211 \\
\hline 118 & 36 & $8: 41: 04.0$ & $-48: 04: 15.6$ & F9 & 3 & 13.31 & 0.61 & $6.6 \pm 5.1$ & $5.8 \pm 5.1$ & $\cdots$ \\
\hline 119 & 41 & $8: 41: 13.7$ & $-48: 04: 36.7$ & F5 & 3 & 12.77 & 0.49 & $-7.2 \pm 3.6$ & $1.5 \pm 1.4$ & $\cdots$ \\
\hline 100 & 45 & $8: 41: 19.2$ & $-48: 13: 04.2$ & & $\ldots$ & 14.32 & 0.68 & $\ldots$ & $\ldots$ & $\ldots$ \\
\hline 128 & $\ldots$ & $8: 41: 30.4$ & $-47: 58: 41.5$ & B9IV & 2 & 9.92 & -0.04 & $-1.2 \pm 2.1$ & $6.7 \pm 1.3$ & L57, HD 74338 \\
\hline 129 & $\ldots$ & $8: 41: 35.3$ & $-47: 57: 51.3$ & $\mathrm{~A} 1$ & 3 & 11.12 & 0.09 & $-2.4 \pm 1.9$ & $2.4 \pm 1.4$ & CD-474225 \\
\hline 50 & 60 & $8: 41: 35.8$ & $-48: 04: 24.1$ & A3 & 3 & 11.30 & 0.12 & $-2.9 \pm 3.0$ & $0.5 \pm 1.4$ & L54 \\
\hline 220 & 63 & $8: 41: 37.3$ & $-48: 20: 47.8$ & $\mathrm{G} 2$ & 3 & 13.03 & 0.64 & $-8.0 \pm 5.8$ & $7.2 \pm 5.8$ & $\cdots$ \\
\hline 41 & 77 & $8: 41: 44.9$ & $-48: 09: 35.8$ & F0 & 3 & 12.17 & 0.38 & $-4.6 \pm 2.2$ & $2.2 \pm 1.4$ & L51 \\
\hline 40 & 83 & $8: 41: 50.5$ & $-48: 09: 17.6$ & F6 & 3 & 13.20 & 0.45 & $1.7 \pm 3.0$ & $-1.7 \pm 2.7$ & L50 \\
\hline 39 & 92 & $8: 41: 55.2$ & $-48: 09: 12.2$ & A3 & 3 & 11.49 & 0.15 & $-6.0 \pm 1.4$ & $4.4 \pm 1.4$ & CD-474238 \\
\hline 266 & 96 & $8: 41: 55.7$ & $-47: 52: 12.6$ & B9/A0V & 2 & 9.84 & -0.02 & $-4.9 \pm 1.7$ & $5.1 \pm 1.8$ & HD 74402 \\
\hline 263 & 107 & $8: 41: 59.5$ & $-47: 48: 17.7$ & B9V & 1 & 10.29 & -0.05 & $-4.6 \pm 1.3$ & $3.4 \pm 1.5$ & CD-474240 \\
\hline 78 & 114 & $8: 42: 04.9$ & $-48: 11: 43.3$ & $\mathrm{~A} 3$ & 3 & 11.88 & 0.17 & $-4.3 \pm 1.4$ & $5.5 \pm 2.3$ & L49 \\
\hline 83 & 121 & $8: 42: 07.5$ & $-48: 14: 40.9$ & $\mathrm{~B} 3 \mathrm{~V}$ & 2 & 8.23 & -0.06 & $-4.1 \pm 1.2$ & $4.4 \pm 1.5$ & L6, HD $74436^{\mathrm{d}}$ \\
\hline 80 & 122 & $8: 42: 08.2$ & $-48: 13: 16.5$ & F7 & 3 & 13.12 & 0.58 & $-6.1 \pm 5.8$ & $2.8 \pm 5.8$ & $\cdots$ \\
\hline 216 & 124 & $8: 42: 09.5$ & $-48: 20: 56.1$ & F3 & 3 & 12.68 & 0.44 & $-4.9 \pm 5.8$ & $8.1 \pm 5.8$ & $\cdots$ \\
\hline 5 & 131 & $8: 42: 11.8$ & $-48: 06: 33.0$ & B9: & 2 & 10.39 & 0.00 & $-3.4 \pm 1.4$ & $4.7 \pm 2.0$ & L43, CD-474247 \\
\hline 38 & 137 & $8: 42: 12.9$ & $-48: 09: 34.5$ & G1 & 3 & 13.60 & 0.66 & $\cdots$ & $\cdots$ & $\cdots$ \\
\hline 6 & 138 & $8: 42: 14.3$ & $-48: 06: 55.9$ & B9 & 3 & 11.28 & 0.09 & $-4.4 \pm 1.4$ & $4.0 \pm 1.4$ & CPD-472576, L44 \\
\hline 211 & 139 & $8: 42: 14.4$ & $-48: 27: 09.2$ & B5V & 2 & 9.46 & -0.04 & $-1.5 \pm 1.7$ & $0.8 \pm 1.2$ & HD 74456 \\
\hline 2 & 142 & $8: 42: 15.8$ & $-48: 04: 20.0$ & A5 & 3 & 11.70 & 0.29 & $-2.2 \pm 3.2$ & $5.3 \pm 2.4$ & L18 \\
\hline 1 & 143 & $8: 42: 16.2$ & $-48: 05: 56.7$ & $\mathrm{~B} 1.5 \mathrm{Vn}$ & 2 & 5.49 & -0.18 & $-3.8 \pm 0.9$ & $2.7 \pm 0.8$ & HD $74455^{\mathrm{e}}, \mathrm{V}^{*} \mathrm{HX} \mathrm{Vel}$ \\
\hline 3 & 148 & $8: 42: 17.8$ & $-48: 03: 53.1$ & B9V & 4 & 10.74 & 0.03 & $-6.8 \pm 2.5$ & $1.1 \pm 1.4$ & L15, CD-474253 \\
\hline 135 & 171 & $8: 42: 26.4$ & $-47: 58: 22.1$ & A6 & 3 & 12.49 & 0.32 & $-4.6 \pm 1.4$ & $7.2 \pm 1.6$ & L20 \\
\hline 57 & 180 & $8: 42: 30.1$ & $-47: 59: 17.9$ & F9 & 3 & 13.29 & 0.63 & $-7.0 \pm 2.7$ & $8.7 \pm 2.5$ & $\cdots$ \\
\hline 35 & 188 & $8: 42: 33.5$ & $-48: 10: 27.8$ & A4 & 3 & 11.91 & 0.22 & $-5.9 \pm 2.3$ & $6.9 \pm 2.3$ & $\cdots$ \\
\hline 34 & $\cdots$ & $8: 42: 34.8$ & $-48: 09: 48.9$ & B2V: & 2 & 7.27 & -0.16 & $-3.7 \pm 0.8$ & $2.9 \pm 0.7$ & L3, HD 74531 \\
\hline 136 & 193 & $8: 42: 35.2$ & $-47: 55: 47.4$ & B8 & 3 & 11.02 & 0.06 & $-6.4 \pm 1.7$ & $3.7 \pm 1.4$ & CD-474260 \\
\hline 17 & 195 & $8: 42: 36.5$ & $-48: 04: 30.9$ & B3IV-V & 2 & 8.71 & -0.11 & $-5.3 \pm 1.7$ & $4.4 \pm 1.6$ & L8, HD 74530 \\
\hline 18 & 189 & $8: 42: 36.6$ & $-48: 05: 04.1$ & B8 & 3 & 10.61 & 0.03 & $-9.4 \pm 2.7$ & $0.8 \pm 3.2$ & L34, CD-474262 \\
\hline 21 & 200 & $8: 42: 37.0$ & $-48: 08: 12.3$ & A0 & 3 & 11.56 & 0.12 & $-8.3 \pm 2.8$ & $4.7 \pm 1.7$ & L28, CD-474264 \\
\hline 205 & $\cdots$ & $8: 42: 39.5$ & $-48: 23: 59.8$ & G1 & 3 & 13.13 & 0.68 & $-6.3 \pm 5.8$ & $-1.8 \pm 5.8$ & $\cdots$ \\
\hline 25 & 207 & $8: 42: 42.1$ & $-48: 05: 17.0$ & A5 & 3 & 11.07 & 0.15 & $-8.7 \pm 1.7$ & $1.3 \pm 1.4$ & L33,CD-474265 \\
\hline 24 & $\cdots$ & $8: 42: 42.4$ & $-48: 06: 27.2$ & A1 & 3 & 11.17 & 0.10 & $-8.5 \pm 2.7$ & $5.4 \pm 1.7$ & L31, CD-474266 \\
\hline 23 & 209 & $8: 42: 43.0$ & $-48: 07: 55.7$ & A0 & 3 & 11.51 & 0.12 & $-4.0 \pm 1.6$ & $4.6 \pm 1.4$ & L29, CD-474267 \\
\hline 141 & 212 & $8: 42: 44.5$ & $-47: 51: 02.5$ & F6 & 3 & 12.69 & 0.49 & $-9.3 \pm 2.5$ & $6.1 \pm 2.2$ & $\cdots$ \\
\hline 27 & $\cdots$ & $8: 42: 46.4$ & $-48: 05: 25.5$ & B9Ve & 2 & 9.49 & -0.03 & $-2.5 \pm 1.3$ & $1.7 \pm 1.7$ & L11, HD $74559^{\mathrm{d}}$ \\
\hline 29 & 220 & $8: 42: 47.2$ & $-48: 08: 04.2$ & $\mathrm{~F} 4$ & 3 & 12.69 & 0.54 & $-9.1 \pm 5.1$ & $13.3 \pm 5.1$ & L26 \\
\hline 73 & 221 & $8: 42: 47.9$ & $-48: 13: 31.9$ & $\mathrm{~B} 6 / 8 \mathrm{~V}$ & 2 & 9.25 & -0.09 & $-4.5 \pm 1.3$ & $1.9 \pm 1.9$ & L21, HD 74581 \\
\hline 74 & 222 & $8: 42: 48.0$ & $-48: 13: 40.4$ & $\mathrm{~B} / \mathrm{A}$ & 2 & 9.93 & 0.02 & $-4.0 \pm 1.5$ & $-1.9 \pm 2.4$ & $\cdots$ \\
\hline 31 & 228 & $8: 42: 53.1$ & $-48: 07: 41.2$ & B3V & 2 & 8.66 & -0.03 & $-1.5 \pm 1.4$ & $3.8 \pm 1.2$ & L7, HD 74580 \\
\hline 72 & 242 & $8: 43: 00.2$ & $-48: 13: 06.6$ & $\mathrm{~B} 7 \mathrm{~V}$ & 4 & 10.42 & -0.02 & $-5.9 \pm 1.3$ & $4.8 \pm 1.3$ & L14, CD-474274 \\
\hline 70 & $\cdots$ & $8: 43: 04.3$ & $-48: 11: 03.7$ & $\mathrm{~B} 8 \mathrm{~V}$ & 2 & 8.91 & -0.04 & $-6.0 \pm 0.9$ & $5.1 \pm 0.7$ & L9, HD 74621 \\
\hline 268 & 255 & $8: 43: 06.5$ & $-48: 18: 16.2$ & G6 & 3 & 14.52 & 0.70 & $-6.9 \pm 5.9$ & $5.3 \pm 5.8$ & $\cdots$ \\
\hline 202 & $\cdots$ & $8: 43: 18.8$ & $-48: 20: 42.9$ & $\mathrm{~B} 3 \mathrm{~V}$ & 2 & 8.87 & -0.11 & $-5.7 \pm 1.3$ & $2.6 \pm 1.1$ & HD 74662 \\
\hline 67 & 277 & $8: 43: 29.1$ & $-48: 02: 54.9$ & $\mathrm{~A} 1$ & 3 & 10.99 & 0.04 & $-4.0 \pm 1.4$ & $3.1 \pm 1.5$ & CD-474286 \\
\hline 175 & 283 & $8: 43: 41.3$ & $-48: 09: 33.1$ & $\mathrm{~F} 3$ & 3 & 12.84 & 0.48 & $-5.9 \pm 2.9$ & $2.6 \pm 2.6$ & $\cdots$ \\
\hline 163 & 287 & $8: 44: 07.8$ & $-48: 01: 14.6$ & A7V & 1 & 11.80 & 0.18 & $-4.9 \pm 1.8$ & $4.4 \pm 2.8$ & CD-474298 \\
\hline 180 & 288 & 8:44:11.4 & $-48: 10: 44.4$ & F8 & 3 & 13.06 & 0.62 & $-8.0 \pm 5.8$ & $1.1 \pm 5.8$ & $\cdots$ \\
\hline 192 & $\cdots$ & $8: 44: 26.6$ & $-48: 27: 05.7$ & A5III & 1 & 11.64 & 0.24 & $-6.3 \pm 1.4$ & $7.5 \pm 3.3$ & CD-474307 \\
\hline
\end{tabular}

Notes. (i) Celestial coordinates are from 2MASS; (ii) optical photometry from Claria et al. (2003); uncertainties are described therein; (iii) proper motions from NOMAD; Zacharias et al. (2004); (iv) mean cluster proper motion derived in Section 3.1.1 is $\mu_{\alpha} \cos \delta=-3.9 \pm 0.4 \mathrm{mas}^{-1}$ and $\mu_{\delta}=3.0 \pm 0.4 \mathrm{mas}^{-1}$. References for spectral type (SpT): (1) Pickles \& Depagne (2010), (2) Skiff (2014), (3) from photometry (see text), (4) from H $\beta$ photometry (Paunzen 2015) calibrated through (Crawford \& Mander 1966).

${ }^{\mathrm{a}}$ From Claria et al. (2003).

${ }^{\mathrm{b}}$ If no cross-reference to Table 4 is provided, we do not confirm the membership assignment.

${ }^{c} \mathrm{CD}$ or CPD—photographic plates from the Annals of the Cape Observatory, L-Lynga, HD—Henry Draper.

${ }^{\mathrm{d}}$ Probable binary.

e Likely ellipsoidal variable (Morris 1985), hence binary. 
computed trend lines of $V$ versus $V-J$ and $V-K$. The scatter was similar (in all these evaluations, we rejected outliers in similar numbers- 8\% - for both bands). However, when the scatter was weighted by the expected $V-J$ or $V-K$ value to create a metric for the uncertainty in stellar type that would result, the metric was a factor of 1.39 smaller for $V-K$. That is, the longer-wavelength baseline resulted in a significant advantage to the use of $V-K$. After identifying the Class II sources, we also tested whether $K$ or IRAC1 (hereafter [3.6]) could be contaminated by excess emission. To do so, we took all of the Class II sources and compared their observed $K-$ [3.6] colors with those we would expect from the spectral types we assigned them as discussed in the following paragraph and using the standard colors of Luhman et al. (2010) for young stars. There were no significant discrepancies, and the average was -0.01 , that is the $K-[3.6]$ color was 0.01 bluer than expected for the standard colors.

Therefore, for types earlier than $\mathrm{K} 4$, we made the type estimates based on the (extinction-corrected) $V-K$ color compared with the tabulation in Mamajek (2015), while for K4 and later we used the $J-[3.6]$ colors from Luhman et al. (2010). After a preliminary assignment of types, we determined empirical loci for the apparent $V$ and [3.6] magnitudes versus type. We rejected any type estimates for stars that deviated from these loci by more than 1.1 magnitudes in $V$ or 0.8 magnitudes in [3.6]. That is, the types were accepted only if the stars were consistent in $V-K$ or $J-[3.6]$ and had apparent magnitudes consistent with cluster membership at both $V$ and [3.6]. Of the 295 members, 80 failed the tests for a consistent classification, of which 13 are Class II sources (see below).

\subsection{Age}

We have put the age of the cluster on the revised age scale (e.g., Bell et al. 2013). To do so, we estimated the cluster age and distance from the catalog of Claria et al. (2003) of probable luminous members, using the main-sequence interior models of Ekström et al. (2012) including the effects of rotation. Given that the photometry is in the plane of apparent color versus apparent magnitude, it is first necessary to transform the interior models into color-magnitude space and then also redden the model corresponding to $E(B-V)=0.09$ mag. To transform the interior models we used the so-called ODFnew models of Castelli \& Kurucz (2004). To redden the model isochrones we used the standard relation $A_{V}=3.1 E(B-V)$ (as appropriate for the low level of reddening, see, e.g., Olson 1975). The most massive star, HD 74455, provides the best age diagnostic for this method. It sits in the vertical region of the model isochrones, hence the small dependences of the estimated age on the color and reddening combined with the small reddening of the cluster itself will have an insignificant effect on the result. This star is a likely ellipsoidal variable (Morris 1985), i.e., a binary, but given the vertical isochrones the single-star luminosity for one of the pair is still compatible with our assigned age. Using main-sequence fitting, we estimated a distance modulus of $\sim 9.5 \mathrm{mag}$ (equivalent to $\sim 800 \mathrm{pc}$; as also found by Claria et al.) and an age of $\sim 9 \mathrm{Myr}$.

This age is confirmed by the $V$ versus $V-J \mathrm{H}-\mathrm{R}$ (CM) diagram in Figure 5, which is primarily based on the low-mass cluster members identified in our study. Both age determinations agree on $~ 9 \mathrm{Myr}$. As is usually the case, the revised calibration gives a significantly older age than the traditional estimate of $6 \pm 2$ Myr (Claria et al. 2003).
IC 2395 is in an age range where absolute ages are not well determined but relative ones are better understood (Soderblom et al. 2014, pp. 219-241). We assign an error of 3 Myr, i.e., we take the age to be $9 \pm 3 \mathrm{Myr}$. This error is to be understood as a statement that IC 2395 is likely to be similar in age to Upper Sco and Ori OB1b, nominally near $10 \mathrm{Myr}$ (on the revised age scale), and neither so young as classic star-forming clusters such as $\rho$ Oph, NGC 1333, NGC 2244, or IC 348 nor so old as Ori OB 1a and Lower Centaurus Crux (LCC)/Upper Centaurus Lupus (UCL). On the traditional age scale, these clusters/ associations are in the same relative sequence, but all at younger ages.

\section{ANALYSIS}

The key result of this section is the identification of candidate IC 2395 cluster members with evidence of circumstellar disks. Spitzer photometry is efficient in identifying evolutionary stages for disks around young stars (e.g., Allen et al. 2004, 2007; Megeath et al. 2004, 2005; Hartmann et al. 2005; Lada et al. 2006; Sicilia-Aguilar et al. 2006; Wang \& Looney 2007). For the youngest systems, these studies have distinguished deeply embedded protostars (Class I) from accreting T Tauri-like stars (Class II) from "normal" stars (Class III), based on placement on IRAC CC diagrams (e.g., [3.6] - [4.5] versus [5.8] - [8.0]). We build on this body of experience to separate Class II sources from the non- or weakexcess Class III cluster members and to identify the transitional disks caught in the process of transformation between these classes. At the age of IC 2395, second-generation debris disks are also starting to appear-these are systems where the dust is not primordial, but is generated in planetesimal collisions.

We have matched the selected cluster members presented in Tables 2 and 3 to the IRAC and MIPS photometry using a radial positional threshold of 2 ". 5 . Several of the objects of Claria et al. (2003) are outside the areas covered with IRAC and MIPS, and a handful are incomplete in the IRAC detections. Altogether 277 objects out of 297 are detected in all four IRAC bands, and 67 of those also have counterparts at $24 \mu \mathrm{m}$. We use this body of photometry to identify the transitional, Class II, and strong debris disks in IC 2395. We find 18 Class II sources $(6.5 \%$ of the sources detected in all four IRAC bands), eight transitional disks $(2.9 \%$ of the full IRAC detections), and 23 debris disk candidates (8.3\%).

The most significant risk in using the longer-wavelength Spitzer channels for identifying stars and protostars with strong infrared excess emission is contamination from thermal dust continuum from the residual natal molecular cloud, including emission from polycyclic aromatic hydrocarbon molecules (PAHs, which contribute strongly at $8 \mu \mathrm{m}$ ), and confusion with background sources along the line of sight. After identifying the cluster members with strong emission from circumstellar disks, we discuss the extent to which contamination may influence these results.

\subsection{Identification of Excess Types among Cluster Members}

Figures 6 and 7 show the objects with excesses on different optical-near-infrared-mid-infrared CM and CC diagrams. The locations of the identified sources are also shown in Figure 2. Two objects (\#6 and \#21) that have large $K-[24]$ excess are not classified because all of our classification schemes require IRAC data and these two stars are outside the area covered by 
Table 3

Optical Photometry of Cluster Members

\begin{tabular}{|c|c|c|c|c|c|c|c|c|c|c|c|c|c|}
\hline ID & $\begin{array}{l}\text { R.A. (2000) } \\
\text { (deg) }\end{array}$ & $\begin{array}{c}\text { Decl. (2000) } \\
\text { (deg) }\end{array}$ & $\mathrm{SpT}^{\mathrm{a}}$ & $\begin{array}{c}U \\
(\mathrm{mag})\end{array}$ & $\begin{array}{l}\text { Error } \\
(\mathrm{mag})\end{array}$ & $\begin{array}{c}B \\
(\mathrm{mag})\end{array}$ & $\begin{array}{l}\text { Error } \\
\text { (mag) }\end{array}$ & $\begin{array}{c}V \\
(\mathrm{mag})\end{array}$ & $\begin{array}{l}\text { Error } \\
\text { (mag) }\end{array}$ & $\begin{array}{c}R \\
(\mathrm{mag})\end{array}$ & $\begin{array}{l}\text { Error } \\
\text { (mag) }\end{array}$ & $\begin{array}{c}I \\
(\mathrm{mag})\end{array}$ & $\begin{array}{l}\text { Error } \\
\text { (mag) }\end{array}$ \\
\hline 1 & 130.04111 & -48.13267 & A1 & 11.463 & $\ldots$ & 11.251 & $\ldots$ & 11.114 & $\ldots$ & $\ldots$ & $\ldots$ & $\cdots$ & $\cdots$ \\
\hline 2 & 130.07856 & -48.49538 & A1 & 11.602 & $\cdots$ & 11.32 & $\cdots$ & 11.116 & $\cdots$ & $\cdots$ & $\cdots$ & $\cdots$ & $\cdots$ \\
\hline 3 & 130.10667 & -48.38002 & F7 & 13.549 & $\ldots$ & 13.381 & $\ldots$ & 12.783 & $\ldots$ & $\ldots$ & $\ldots$ & $\ldots$ & $\ldots$ \\
\hline 4 & 130.13353 & -48.15396 & $\cdots$ & $\ldots$ & $\ldots$ & $\ldots$ & $\ldots$ & $\ldots$ & $\ldots$ & $\cdots$ & $\ldots$ & $\ldots$ & $\cdots$ \\
\hline 5 & 130.16329 & -48.35785 & K8 & 17.685 & 0.014 & 17.052 & 0.009 & 15.836 & 0.011 & 15.122 & 0.012 & 14.41 & 0.022 \\
\hline
\end{tabular}

Note. 2MASS celestial coordinates are used except for ID 110 where IRAC coordinates are used.

a All spectral types in this table are estimated from photometry (see text for details).

(This table is available in its entirety in machine-readable form.)

IRAC. Based on their $K-$ [24] color they can be either transitional disks or class II sources. The identification of the other objects is explained in the next three subsections. Since we will use identical criteria for a sample of 19 clusters and associations as listed in Table 6, we discuss our approach in this general context.

\subsubsection{Transitional Disks}

Although it is desirable to identify transitional disks through infrared spectroscopy (Espaillat et al. 2014), our aim is for a large sample to investigate their incidence and how it evolves with age. Since infrared spectroscopy is not available for all of this sample, we used photometry to test for photospheric-like colors in the $4 \mu \mathrm{m}$ region and to measure the size of the excess at $24 \mu \mathrm{m}$, and also to impose a requirement on the spectral type of the star for systems old enough that extreme debris disks might be confused with transitional ones.

We first derive a criterion to test for photospheric-like colors. We start with a simple physical definition, obtained from Sicilia-Aguilar et al. (2008): a transitional disk should have no excess out to $6 \mu \mathrm{m}$, but should retain a large excess at $24 \mu \mathrm{m}$, indicative of retention of much of the primordial disk in the more distant zone that dominates at this wavelength. In this regard, transitional disks can be distinguished from Class II sources, which have significant excesses already at $6 \mu \mathrm{m}$ (Sicilia-Aguilar et al. 2008). The simplest way to isolate candidate transitional disks, then, is to use a color difference involving IRAC band 3 at $5.8 \mu \mathrm{m}$. We prefer [3.6] - [5.8] because we will want to apply identical criteria to many clusters and associations, some with significant reddening, and this color difference is significantly less affected by extinction than is the case for color differences involving shorter wavelengths, such as $K$ (e.g., Flaherty et al. 2007). In addition, the measurements of [3.6] and [5.8] are obtained at the same time and hence are not affected by variability.

To determine the acceptable values of [3.6] - [5.8] to isolate candidate transitional disks from Class II sources, we show in Figure 8 the distribution of this color difference for our entire sample of stellar clusters and associations (listed in Table 6), along with a Gaussian fit to the primary peak in the distribution. There is a distinct excess over the Gaussian fit for sources with [3.6] $-[5.8]<0.4$. We adopt this value as the limit for a candidate transitional disk since it defines a class of object that apparently does not belong to the population of typical Class II young stellar objects (YSOs). The $K-[6 \mu \mathrm{m}]$ slope quoted as the upper limit for transitional disks by Kim et al. (2013) predicts this same color, while the limits found by Espaillat et al. (2014, pp. 497-520) and Muzerolle et al. (2010) are more lenient, equivalent respectively to [3.6] $-[5.8]=0.48$ and 0.56 .

In the cases of Upper Sco, LCC, UCL, and TW Hya, we have identified transitional disks from WISE photometry. To determine a criterion similar to the one we have adopted for IRAC photometry, we compared $W 1-W 2$ versus [3.6] - [5.8] for more than 100 sources with measurements in both systems in Upper Sco, finding that the equivalent limit is $W 1-W 2<0.43$ with a nominal error of 0.01 .

We also need to define a minimum level of excess at $24 \mu \mathrm{m}$. Muzerolle et al. (2010) define "weak excess" transitional disks with a slope equivalent to [8]-[24] $\geqslant 1.5$. They also define an optically thick disk with a slope roughly equivalent to [8] $[24]=3.5$. We adopt a threshold of [8] $-[24]=1.5$ and compare our results at this level with those at [8] - [24] $>2.5$ and $[8]-[24]>3.5 .^{15}$ To determine similar criteria for measurements with WISE, we used measurements of sources in Upper Sco detected in both sets of photometry to set the equivalent threshold to be $W 3-W 4>0.8$ and $W 2-W 4>1.7$.

A question remains of whether the Spitzer measurements should also put a limit on how strong the source infrared excess can be at IRAC4 $(8 \mu \mathrm{m})$. We explored the implications of strong fluxes in this band using the YSO disk SED-fitting tool (Robitaille et al. 2006) and found that disks with masses and accretion rates well within the range expected for transitional disks (e.g., disk masses of $\sim 10^{-6} M_{\odot}$ and accretion $<10^{-10}$ $M_{\odot} \mathrm{yr}^{-1}$ ) could have substantial fluxes at $8 \mu \mathrm{m}$. In addition, the broad IRAC $8 \mu \mathrm{m}$ band can include silicate emission, which can be strong in transitional disks. Therefore, we imposed no requirement there.

Finally, we imposed a spectral type criterion. The great majority of transitional disks are around stars of spectral type later than F (Muzerolle et al. 2010; Espaillat et al. 2014, pp. 497-520). The evolution of protoplanetary disks is faster around higher-mass stars (e.g., Kennedy \& Kenyon 2014; Yasui et al. 2014). Therefore, by an age of $\sim 10 \mathrm{Myr}$, we do not expect to find many transitional disks around early-type stars. To reflect this trend, we imposed the requirement that the stellar spectral type for ages $>6 \mathrm{Myr}$ had to be later than F (either from spectroscopy or estimated through photometry) to accept a disk as being transitional. The transitional disks in IC 2395 resulting from these criteria are indicated in Table 5 (WT for weak transitional, [8] - [24] $<2.5$, and $\mathrm{T}$ for transitional). Our final selection criteria are also illustrated in Figure 9.

\footnotetext{
${ }^{15}$ For comparison, Cieza et al. (2012) use a roughly similar threshold of [3.6] $-[24]=1.5$ to identify candidate transitional disks.
} 
Table 4

Infrared Photometry of Cluster Members

\begin{tabular}{|c|c|c|c|c|c|c|c|c|c|c|c|c|c|c|c|c|}
\hline ID & $\begin{array}{c}J^{\mathrm{a}} \\
(\mathrm{mag})\end{array}$ & $\begin{array}{l}\text { Error } \\
\text { (mag) }\end{array}$ & $\begin{array}{c}H^{\mathrm{a}} \\
(\mathrm{mag})\end{array}$ & $\begin{array}{l}\text { Error } \\
\text { (mag) }\end{array}$ & $\begin{array}{c}K_{S}^{\mathrm{a}} \\
(\mathrm{mag})\end{array}$ & $\begin{array}{l}\text { Error } \\
\text { (mag) }\end{array}$ & $\begin{array}{l}{[3.6]} \\
\text { (mag) }\end{array}$ & $\begin{array}{l}\text { Error } \\
\text { (mag) }\end{array}$ & $\begin{array}{l}\text { [4.5] } \\
(\mathrm{mag})\end{array}$ & $\begin{array}{l}\text { Error } \\
\text { (mag) }\end{array}$ & $\begin{array}{l}{[5.8]} \\
\text { (mag) }\end{array}$ & $\begin{array}{l}\text { Error } \\
\text { (mag) }\end{array}$ & $\begin{array}{l}{[8.0]} \\
\text { (mag) }\end{array}$ & $\begin{array}{l}\text { Error } \\
\text { (mag) }\end{array}$ & $\begin{array}{l}{[24]} \\
\text { (mag) }\end{array}$ & $\begin{array}{l}\text { Error } \\
\text { (mag) }\end{array}$ \\
\hline 1 & 10.931 & 0.024 & 10.887 & 0.021 & 10.87 & 0.021 & $\ldots$ & $\ldots$ & 10.86 & 0.004 & $\ldots$ & $\ldots$ & 10.729 & 0.012 & $\ldots$ & $\ldots$ \\
\hline 2 & 10.861 & 0.023 & 10.837 & 0.024 & 10.81 & 0.019 & $\cdots$ & $\ldots$ & $\cdots$ & $\ldots$ & $\cdots$ & $\ldots$ & $\ldots$ & $\cdots$ & $\cdots$ & $\cdots$ \\
\hline 3 & 11.812 & 0.027 & 11.466 & 0.024 & 11.414 & 0.023 & $\cdots$ & $\cdots$ & $\cdots$ & $\cdots$ & $\cdots$ & $\cdots$ & $\cdots$ & $\cdots$ & $\cdots$ & $\cdots$ \\
\hline 4 & 15.07 & 0.049 & 14.308 & 0.048 & 13.813 & 0.052 & 13.351 & 0.01 & 13.175 & 0.012 & 13.09 & 0.043 & 12.38 & 0.044 & $\cdots$ & $\ldots$ \\
\hline 5 & 13.304 & 0.024 & 12.776 & 0.029 & 12.592 & 0.026 & $\cdots$ & $\cdots$ & 12.601 & 0.009 & $\cdots$ & $\cdots$ & 12.491 & 0.044 & $\cdots$ & $\cdots$ \\
\hline
\end{tabular}

Note.

${ }^{\text {a }} J H K$ photometry from 2MASS.

(This table is available in its entirety in machine-readable form.)

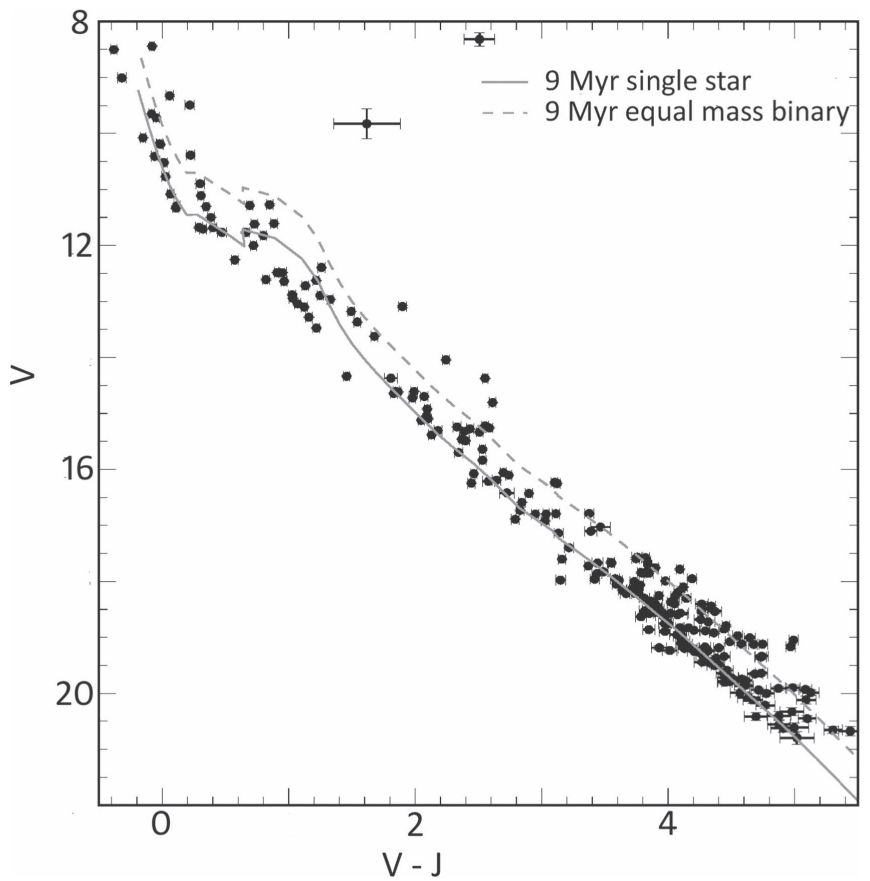

Figure 5. $V$ vs. $V-J$ CM diagram with semi-empirical (see Bell et al. (2014) for details) model isochrones using the Dartmouth interior models. The red solid isochrone is for $9 \mathrm{Myr}$; it has been reddened by $E(B-V)=0.09$ and shifted in distance assuming a modulus of 9.5 . The dashed line is the same but shifted 0.75 mag higher, i.e., where we would expect the equal-mass binaries to lie.

To test the degree of debris-disk contamination in these selections, we used the list of 10 extreme debris systems in Balog et al. (2009) (we excluded HD 21362 since its excess is dominated by free-free emission). These sources have excesses at $24 \mu \mathrm{m}$ by at least a factor of four (i.e., $1.5 \mathrm{mag}$, the threshold for our identifying a weak transitional disk) and hence can be compared directly with our candidate transitional disks, since the latter are required to have similar excesses. We used the identical approaches with these sources, first testing with Spitzer [3.6] [5.8] photometry (from Gorlova et al. 2007; Balog et al. 2009; Weinberger et al. 2011), and if that was lacking, then using WISE measurements. Two of the extreme debris systems are too red in these colors to pass our transitional disk criterion; if we also impose the spectral type criterion, only BD 20307 would pass our selection. Only HR 4796A has [8] - [24] $>2.5$, but it is too early-type to satisfy our criteria. Therefore, the potential contamination appears to be small, particularly at the higher thresholds for $24 \mu \mathrm{m}$ excess. If the threshold [8] - [24] $>1.5$ were allowing a significant number of extreme debris disks, we
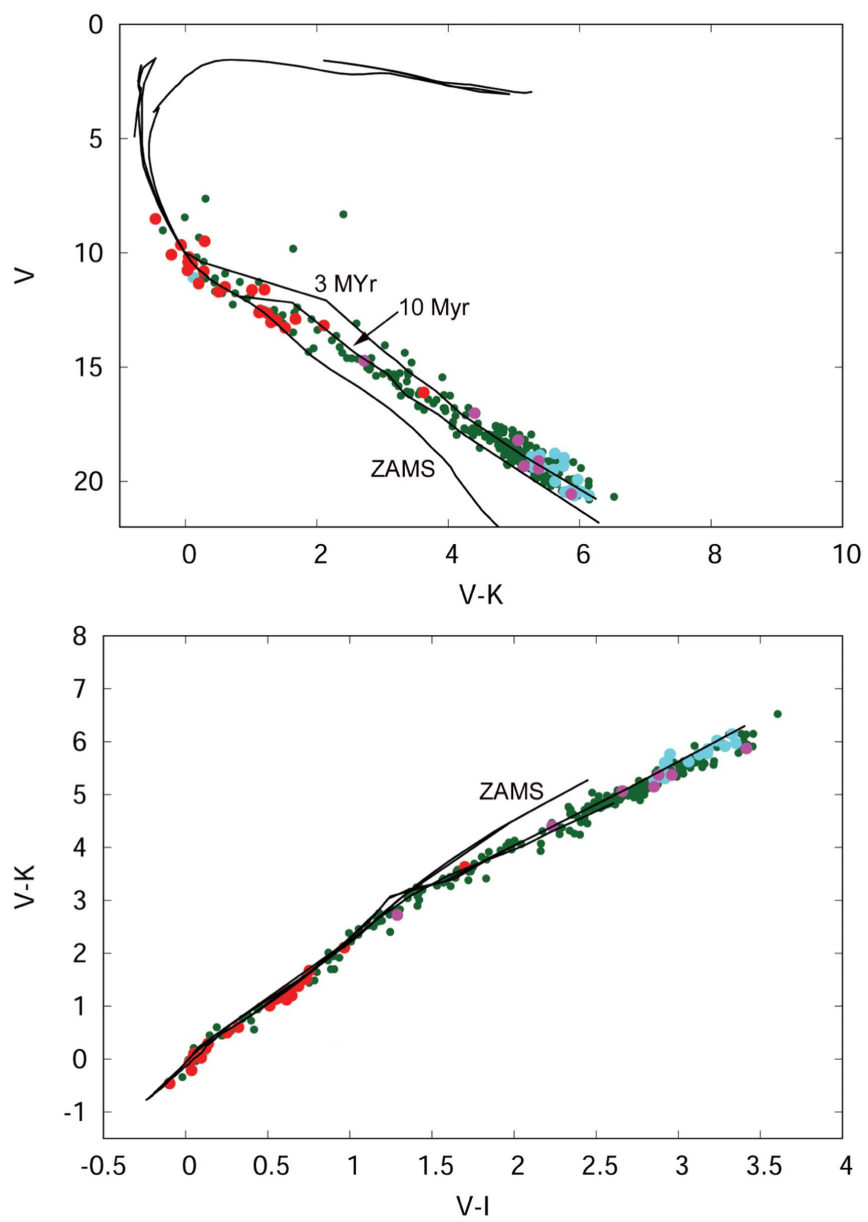

Figure 6. Optical-near-infrared CM and CC diagrams of IC 2395 cluster members. Green dots: members without IR excess, red: debris disk candidates, magenta: transitional disk candidates, cyan: class II candidates. Black lines show the 10 and 3 Myr isochrones (Palla \& Stahler 1999) (traditional age scale) and the ZAMS (Marigo et al. 2008).

would expect the fraction of transitional disk candidates to decrease as the threshold was increased, but Table 6 shows that, if anything, there is a trend in the opposite direction. We conclude that, at least for the samples $\leqslant 15 \mathrm{Myr}$ in age, debrisdisk contamination is not an issue.

\subsubsection{Class II Objects}

Class II objects are pre-main-sequence stars characterized by SEDs with photospheric emission at visible wavelengths 


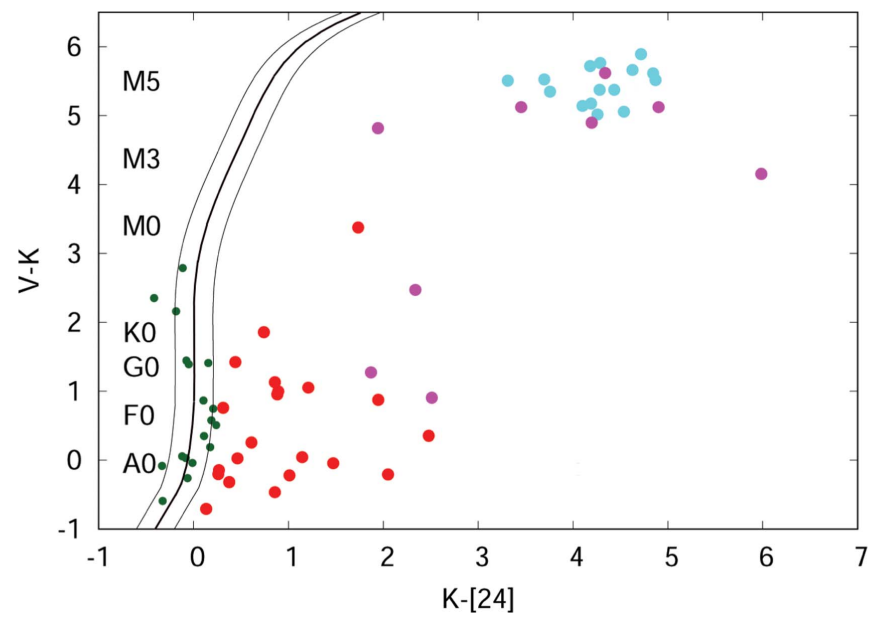

Figure 7. Dereddened $V-K$ vs. $K_{s}-$ [24.0] CC diagram. Symbols are as in Figure 6 . The black line represents the photospheric colors of main-sequence stars (Urban et al. 2012), while the bounding lines are the typical range of uncertainties in projecting the color from shorter wavelengths (including random and systematic errors).

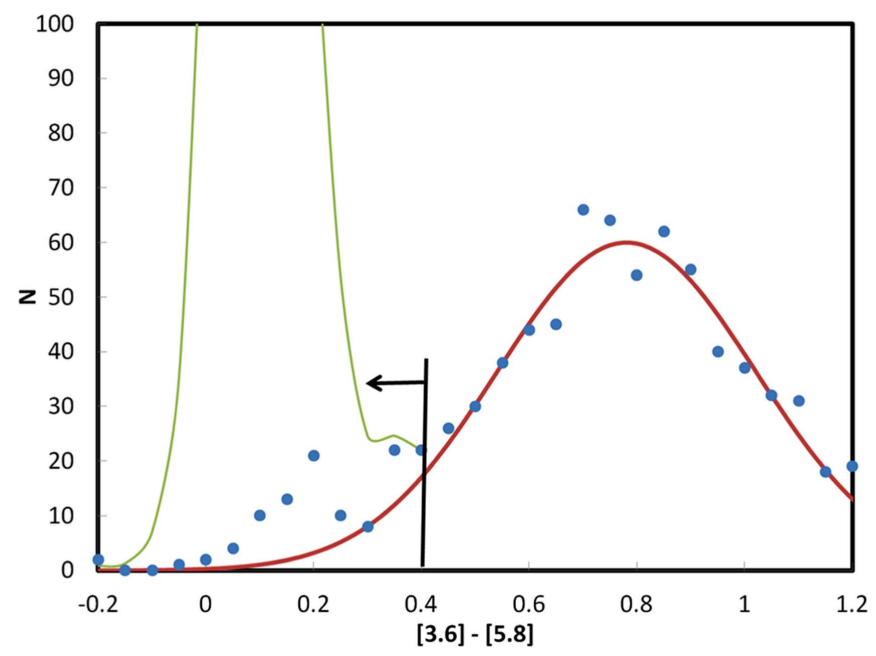

Figure 8. Selection criterion for transitional disks using IRAC colors. The points show the distribution of the colors for all the objects with [8] [24] $>1.5$ in all the clusters under study (see text), to which we have fitted a Gaussian (solid red line) confined to the upper two-thirds of the points to avoid biasing the fit with the wings of the distribution. The thin green line shows the distribution for the sources with smaller [24] excess emission. The vertical line shows the criterion adopted to identify transitional disk candidates (i.e., [3.6] $-[5.8]<0.4)$

up to about $2 \mu \mathrm{m}$ followed by flat or gradually decreasing slopes at longer wavelengths (e.g., Lada \& Wilking 1984; Lada et al. 1987). The mid-infrared emission is believed to be due to large amounts of heated dust dispersed in an optically thick, gas-rich primordial disk. In some cases, there may also be an ultraviolet excess component of the SED, indicating radiation emitted from an accretion shock on the star's surface (Muzerolle et al. 2003). Class II includes classic T Tauri stars (CTTSs) and Herbig AeBe (HAeBe) stars. Their existence within a cluster, especially in large numbers, implies stellar ages on the traditional scale of less than about 10 Myr (e.g., Haisch et al. 2001; Hillenbrand 2005; Mamajek 2005). Stars older than this have typically dissipated their primordial gas (e.g., Pascucci et al. 2006) so that any thermal dust emission is optically thin.
Our criteria for identifying transitional disks join consistently onto the criteria for photometric identification of Class II sources from Gutermuth et al. (2009), which are: [3.6] $[5.8]>0.4$ and $[4.5]-[8]>0.268 \times([3.6]-[5.8])+0.393$. We also required a detection at 22 or $24 \mu \mathrm{m}$. These objects are designated II in Table 5.

\subsubsection{Debris Disks}

Debris disks represent the final evolutionary state of circumstellar disks. By this stage, the primordial gas has fully dissipated and impacts among the planetesimals create a collisional cascade, resulting in a dusty, gas-less disk. When heated by the central star, the dust reradiates in the midinfrared. Stars in IC 2395 with [8] - [24] > 0.15 and spectral type of F or earlier or with [8] - [24] $<1.5$ and of later spectral type were designated debris disks (D in Table 5). If no spectral type could be assigned photometrically (and none was available spectroscopically), we designate the status in Table 5 as D?. Sources 8 and 15 are two marginal cases with [8] $-[24]=0.15$ at significance levels of $5 \sigma$ and $3.4 \sigma$, respectively. We have not included them in Table 5, but their parameters can be recovered from Tables 3 and 4 if desired. We identified 23 debris disk candidates. A number of the debris disk candidates have modest $(0.15-0.30 \mathrm{mag})$ IRAC band excesses at $8 \mu \mathrm{m}$.

\subsection{Contamination}

The most likely contaminant in our identification of stars with $24 \mu \mathrm{m}$ excesses is confusion from random line-of-sight positional overlap with distant optically faint but infraredbright galaxies, planetary nebulae, and active galactic nuclei. The effects of these contaminations are usually small (Megeath et al. 2004; Gutermuth et al. 2008). For example, with $\sim 2000$ extragalactic $24 \mu \mathrm{m}$ sources per square degree at $0.5 \mathrm{mJy}$ (Papovich et al. 2004), a flux less than our completeness limit but greater than our detection limit, the probability of a chance background source observed within our matching radius of 2.15 of any single cluster member is $0.3 \%\left(\pi\left(2.5^{2} / 3600^{2}\right) \times 2000\right)$, which means that in our sample of almost 300 stars the probability of one chance alignment is close to $100 \%$, but more than a few cases is unlikely. We examined the angular offsets between the 2MASS and the IRAC and MIPS positions of our candidate IR excess stars to evaluate whether their excesses can be attributed to chance alignment. We found that the average offset between the 2MASS and IRAC coordinates is about 0 ". $3 \pm 0$ ". 3 with a maximum separation of 0 .! 68 . That is, the probability of a chance alignment with a $2 \mathrm{MASS}$ source is around $1 \%$. For the objects with MIPS photometry, we found that the average offset between the 2MASS and MIPS coordinates is 0 ." $5 \pm 0$ ". 5 , with a large portion of the error coming from a few sources where the distance is larger than $1^{\prime \prime}$. These are member candidate Nos. 37, 219, 44, 82, 198, 242,230 , and 122 , with offsets respectively of $2 . ! 25,1 . ! 58$,

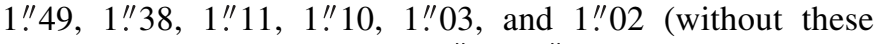
sources the average error is 0 ". $4 \pm 0$ ". 3 with no significant offset). Given the errors, an offset for a single source of $1^{\prime \prime}$ is plausible, while an offset of 1 !' 5 is unlikely but still possible; any larger offset is probably a chance alignment. Therefore, we removed star No. 37 from the sample of infrared excess sources (although its identification as a cluster member is still valid). We visually examined the images of Nos. 219, 44, and 
Table 5

Disk Candidates in IC 2395

\begin{tabular}{|c|c|c|c|c|c|c|c|c|}
\hline ID & $\mathrm{SpT}$ & $\begin{array}{c}{[3.6]-[5.8]} \\
(\mathrm{mag})\end{array}$ & $\begin{array}{l}\text { Error } \\
(\mathrm{mag})\end{array}$ & $\begin{array}{c}{[4.5]-[8]} \\
(\mathrm{mag})\end{array}$ & $\begin{array}{l}\text { Error } \\
(\mathrm{mag})\end{array}$ & $\begin{array}{l}{[8]-[24]} \\
\quad(\mathrm{mag})\end{array}$ & $\begin{array}{l}\text { Error } \\
(\mathrm{mag})\end{array}$ & $\begin{array}{l}\text { Disk } \\
\text { Type }\end{array}$ \\
\hline 14 & $\ldots$ & -0.014 & 0.005 & 0.162 & 0.006 & 1.154 & 0.034 & $\mathrm{D}$ ? \\
\hline 25 & M6 & 0.467 & 0.050 & 0.756 & 0.055 & 2.795 & 0.057 & II \\
\hline 29 & M6 & 0.36 & 0.062 & 0.81 & 0.070 & 3.873 & 0.069 & $\mathrm{~T}$ \\
\hline 36 & F9 & 0.103 & 0.022 & 0.142 & 0.028 & 1.685 & 0.036 & $\mathrm{D}$ ? \\
\hline 41 & F5 & 0.137 & 0.016 & 0.199 & 0.020 & 0.829 & 0.042 & $\mathrm{D}$ \\
\hline 44 & $\ldots$ & 0.096 & 0.040 & 0.099 & 0.058 & 1.694 & 0.092 & WT \\
\hline 63 & $\mathrm{G} 2$ & 0.197 & 0.014 & 0.022 & 0.016 & 0.428 & 0.051 & $\mathrm{D}$ \\
\hline 71 & $\ldots$ & 0.122 & 0.029 & 0.205 & 0.047 & 1.416 & 0.066 & $\mathrm{D} ?$ \\
\hline 80 & $\ldots$ & 0.702 & 0.060 & 0.9 & 0.060 & 3.156 & 0.062 & II \\
\hline 83 & F6 & 0.09 & 0.023 & 0.304 & 0.023 & 0.856 & 0.052 & $\mathrm{D}$ \\
\hline 96 & $\mathrm{~B} 9 / \mathrm{A} 0 \mathrm{~V}$ & 0.183 & 0.007 & -0.041 & 0.007 & 0.455 & 0.019 & $\mathrm{D}$ \\
\hline 102 & M6 & 0.459 & 0.041 & 0.711 & 0.046 & 2.127 & 0.059 & II \\
\hline 107 & B9V & 0.026 & 0.008 & 0.052 & 0.009 & 0.902 & 0.031 & $\mathrm{D}$ \\
\hline 109 & $\ldots$ & 0.598 & 0.080 & 1.014 & 0.062 & 3.211 & 0.062 & II \\
\hline 110 & $\ldots$ & 0.798 & 0.084 & 0.986 & 0.070 & 2.648 & 0.079 & II \\
\hline 122 & F7 & 0.14 & 0.018 & 0.037 & 0.033 & 0.871 & 0.064 & $\mathrm{D}$ \\
\hline 124 & F3 & 0.051 & 0.015 & 0.062 & 0.022 & 2.447 & 0.025 & D \\
\hline 130 & $\ldots$ & 0.618 & 0.027 & 0.888 & 0.037 & 2.63 & 0.039 & II \\
\hline 133 & $\ldots$ & 0.34 & 0.127 & 1.088 & 0.088 & 3.167 & 0.086 & $\mathrm{~T}$ \\
\hline 138 & B9 & 0.438 & 0.010 & 0.881 & 0.008 & 3.026 & 0.010 & II \\
\hline 140 & $\ldots$ & 0.592 & 0.053 & 0.862 & 0.056 & 2.648 & 0.060 & II \\
\hline 142 & A5 & 0.053 & 0.012 & 0.178 & 0.012 & 2.374 & 0.014 & $\mathrm{D}$ \\
\hline 147 & $\ldots$ & 0.551 & 0.074 & 1.044 & 0.087 & 2.983 & 0.089 & II \\
\hline 148 & B9V & 0.068 & 0.009 & 0.036 & 0.011 & 0.281 & 0.029 & $\mathrm{D}$ \\
\hline 151 & $\ldots$ & 0.049 & 0.012 & 0.063 & 0.017 & 0.706 & 0.040 & $\mathrm{D} ?$ \\
\hline 159 & $\cdots$ & 0.379 & 0.104 & 0.95 & 0.087 & 2.897 & 0.090 & $\mathrm{~T}$ \\
\hline 164 & M6 & 0.154 & 0.062 & 0.605 & 0.062 & 3.033 & 0.065 & $\mathrm{~T}$ \\
\hline 166 & $\cdots$ & 0.431 & 0.089 & 1.024 & 0.077 & 3.284 & 0.076 & II \\
\hline 173 & $\cdots$ & 0.624 & 0.063 & 0.861 & 0.098 & 2.822 & 0.102 & II \\
\hline 188 & A4 & 0.055 & 0.015 & -0.001 & 0.024 & 0.765 & 0.061 & $\mathrm{D}$ \\
\hline 189 & $\ldots$ & 0.537 & 0.042 & 0.697 & 0.060 & 2.426 & 0.067 & II \\
\hline 191 & $\mathrm{~F} 1$ & 0.049 & 0.013 & 0.047 & 0.021 & 0.334 & 0.039 & $\mathrm{D}$ \\
\hline 193 & B8 & -0.007 & 0.011 & 0.166 & 0.013 & 0.939 & 0.025 & $\mathrm{D}$ \\
\hline 195 & B3IV-V & 0.011 & 0.004 & -0.019 & 0.005 & 0.228 & 0.025 & $\mathrm{D} ?$ \\
\hline 198 & B8 & 0.058 & 0.010 & 0.231 & 0.012 & 2.022 & 0.015 & $\mathrm{D}$ \\
\hline 200 & $\mathrm{~A} 0$ & 0.04 & 0.013 & 0.006 & 0.039 & 1.512 & 0.045 & $\mathrm{D}$ \\
\hline 213 & $\ldots$ & 0.673 & 0.025 & 1.131 & 0.027 & 2.899 & 0.028 & II \\
\hline 215 & $\cdots$ & 0.019 & 0.052 & 0.612 & 0.048 & 5.289 & 0.046 & $\mathrm{~T}$ \\
\hline 219 & M6 & 0.286 & 0.055 & 0.782 & 0.080 & 2.227 & 0.086 & WT \\
\hline 230 & M6 & 0.593 & 0.046 & 0.906 & 0.096 & 2.673 & 0.097 & II \\
\hline 240 & $\cdots$ & 0.698 & 0.025 & 1.036 & 0.037 & 2.424 & 0.039 & II \\
\hline 242 & B7V & -0.046 & 0.009 & 0.027 & 0.016 & 0.339 & 0.028 & $\mathrm{D}$ \\
\hline 245 & $\ldots$ & 0.492 & 0.040 & 0.73 & 0.048 & 2.664 & 0.051 & II \\
\hline 253 & $\mathrm{~F} 4$ & 0.029 & 0.009 & 0.168 & 0.021 & 0.748 & 0.031 & $\mathrm{D}$ \\
\hline 256 & M6 & 0.433 & 0.070 & 0.977 & 0.052 & 3.118 & 0.069 & II \\
\hline 257 & $\cdots$ & 0.665 & 0.072 & 0.651 & 0.097 & 2.048 & 0.117 & II \\
\hline 277 & A1 & 0.062 & 0.009 & 0.008 & 0.012 & 0.385 & 0.029 & $\mathrm{D}$ \\
\hline 281 & $\mathrm{~K} 4$ & 0.181 & 0.022 & 0.076 & 0.028 & 2.22 & 0.033 & WT \\
\hline 283 & F3 & 0.131 & 0.017 & 0.082 & 0.020 & 1.911 & 0.027 & $\mathrm{D}$ \\
\hline
\end{tabular}

82 and found that No. 82 is close to a bright star in an area of relatively large background confusion, also indicated by its large measurement errors; it also was removed from the infrared excess sample. No. 219 has no obvious background contamination and shows a large excess even in the IRAC bands, which makes it unlikely to be a spurious detection. We are left with No. 44 as the only ambiguous candidate. However, this star is on well-behaved background, so we have accepted it. To summarize, we found two IR excess candidates (Nos. 37 and 82) that are probably contaminated, and we removed them in the final analysis.

\section{DISCUSSION: THE PLACE OF TRANSITIONAL DISKS IN DISK EVOLUTION}

IC 2395 includes a large proportion of transitional disks. Transitional disks were first identified from IRAS data as a class with little or no excess emission at wavelengths short of $10 \mu \mathrm{m}$, indicating little dust close to the star, but with strong excesses at longer wavelengths indicative of an optically thick outer disk (Strom et al. 1989). Multiple interpretations have been advanced for this behavior (Williams \& Cieza 2011). Previous studies have invoked the presence of a planet (Rice et al. 2003; Quillen et al. 2004), dust evolution (Wilner et al. 


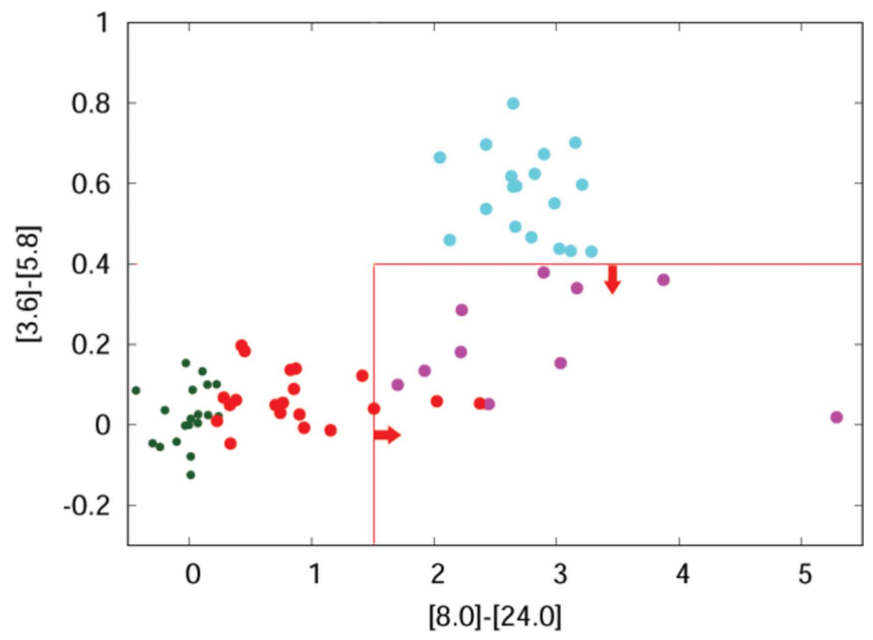

Figure 9. Color-color diagram illustrating the selection criteria (within the red box) for transitional disks (i.e., [3.6] - [5.8] $<0.4$ and [8] $-[24]>1.5$ ) applied to IC 2395. Symbols are as in Figures 6 and 7.

2005), or photoevaporation (Hollenbach et al. 2000; Alexander et al. 2006; Goto et al. 2006) to explain the observed "holes."

With the end of the Spitzer and WISE cryogenic missions, and publication of nearly all the cluster observations obtained with them, the pace of finding major new samples of transitional disks will slow substantially, making an update of their behavior timely. The most recent work along the same lines (Espaillat et al. 2014) utilized Upper Sco as its oldest sample, but since then questions have been raised about the age of the low-mass members of this moving group (Herczeg \& Hillenbrand 2015) that may undermine it as a probe of $11 \mathrm{Myr}$ old transitional disks. IC 2395 (and other stellar clusters/ associations of similar age) can provide a critical independent estimate. In the following four paragraphs, we set the scene for this analysis by discussing age scales and then sample selection. In the next section we show that, in general, the proportion of transitional disks does not change dramatically from one cluster/association to another of similar age. This conclusion justifies our averaging of the transitional disk proportions in the following section, to derive high-weight proportions of transitional disks in young and middle-aged clusters/associations (1-3.5 and 4-6.5 Myr respectively on the traditional calibration, 1-6 and 8-13 Myr on the revised one).

We will focus on the young stellar clusters and associations listed in Table 6, for which we show ages on both the revised and the traditional calibrations. Because ages on the traditional calibration have been determined in a variety of ways, in obtaining a homogeneous set we have given priority to those selected in the review by Mamajek (2009). Where ages on the revised calibration are not available directly, we have assigned them according to similarity in published H-R diagrams for clusters/associations with directly determined ages. These cases are indicated in the notes to the table. As a test of this procedure, we evaluated Ori OB1b, which, according to the $\mathrm{H}$ $\mathrm{R}$ diagram from Hernández et al. (2008), should have an age similar to those of $\lambda$ Ori, NGC 2362, and $\gamma$ Vel, all of which are indicated to be $10-12 \mathrm{Myr}$ on the revised scale. We took the measurements of low-mass members of Ori OB1b from Briceño et al. (2005), adjusted them to the distance of IC 2395, and applied extinction corrections to the individual stars according to the estimates of this parameter in Briceño et al.
(2005). We then superimposed the resulting $V$ versus $V-J$ diagram on the one for IC 2395 shown in Figure 5. The agreement is excellent, indicating an age of $\sim 9 \mathrm{Myr}$ for Ori OB $1 b$, although there is more scatter for the stars in it, presumably because of the complications introduced by the larger and variable extinction. This agreement supports the age assignment on the revised calibration.

Importantly for this study, both the traditional and revised age calibrations place the cluster/associations into age groupings identically, as shown in Table 6. Therefore, our conclusions about the behavior of transitional disks will only change in timescale with a change in age calibration, but otherwise will remain identical.

A critical issue is how, in each case, to define a sample of cluster members for study. The most conservative approach is to require that the members be identified through techniques not involving the mid-infrared (to avoid any possibility of introducing a bias into the infrared characteristics we use to identify transitional disks). We have applied the same criteria for transitional and Class II disks as discussed above for IC 2395. Table 6 lists the ratios of transitional to (transitional + Class II) disks for each stellar cluster or association. It also shows the totals for three age ranges: 1-6, 8-13, and 14-17 Myr (all on the revised calibration, corresponding to 1-3.5, 4-6.5, and 7-16 Myr on the traditional calibration). For the young clusters within $1 \mathrm{kpc}$, the totals extend down to moderately late-type, low-mass stars, while for the two more distant young clusters (NGC 2244 and 2362), the infrared measurements extend down only to intermediate-mass stars. Therefore, we also show the totals omitting the two distant clusters. These values are in boldface because we believe them to be the most reliable indicators of transitional disk behavior. We show similar boldface numbers for the intermediate age range, but both groupings in the oldest age range are closer than $1 \mathrm{kpc}$.

A less conservative approach uses the Spitzer data to identify cluster members, yielding significantly larger numbers in the samples. The results with this approach are also tabulated for the youngest age range ${ }^{16}$; for the intermediate and old ranges, the cluster members are all identified without Spitzer data so this case is not shown. The results for all three levels of conservatism are identical within the errors, indicating that they are relatively robust to the member-identification approach.

\subsection{Does the Proportion of Transitional Disks Vary from Cluster to Cluster?}

Table 6 shows that, in general, the individual clusters/ associations in the young ( $\leqslant 6 \mathrm{Myr}$ on the revised scale) and middle-aged (8-13 Myr) categories have incidences of transitional disks consistent within the errors with the averages for each group (in particular, Upper Sco seems to be in line with other clusters and associations of similar age). This is also true for the old category, but in that case with very minimal counts. To make this comparison quantitative, we use the difference from the average in units of the quoted errors for all the young and middle-aged systems. The result is a nearly perfect normal distribution, except that the number of transitional disks in $\rho$ Oph is low at $2 / 123$ or $1.6 \%$. Turning to the infrared-selected sample, this cluster is still low in transitional disks, $9 / 249$ or $3.6 \%$, but now at less than $3 \sigma$ from the average. That is, there is

\footnotetext{
${ }^{16}$ We do not show the values for the individual clusters.
} 
Table 6

Proportion of Transitional Disk Candidates among Known Members of Clusters and Associations

\begin{tabular}{|c|c|c|c|c|c|c|c|c|c|c|c|c|}
\hline $\begin{array}{l}\text { Cluster or } \\
\text { Association } \\
\text { (1) }\end{array}$ & $\begin{array}{l}\text { Revised Age } \\
\text { (Myr) } \\
\text { (2) }\end{array}$ & $\begin{array}{l}\text { Ref. } \\
\text { age } \\
\text { (3) }\end{array}$ & $\begin{array}{c}\text { Trad. Age } \\
\text { (Myr) } \\
\text { (4) }\end{array}$ & $\begin{array}{l}\text { Ref. } \\
\text { age } \\
(5)\end{array}$ & $\begin{array}{l}\text { Ref. } \\
\text { Mshp } \\
\text { (6) }\end{array}$ & $\begin{array}{c}\text { Trans./Total } \\
{[8]-[24]>1.5} \\
(7)\end{array}$ & $\begin{array}{l}\% \\
(8)\end{array}$ & $\begin{array}{c}\text { Trans./Total } \\
{[8]-[24]>2.5} \\
(9)\end{array}$ & $\begin{array}{l}\% \\
(10)\end{array}$ & $\begin{array}{c}\text { Trans./Total } \\
{[8]-[24]>3.5} \\
(11)\end{array}$ & $\begin{array}{l}\% \\
(12)\end{array}$ & $\begin{array}{c}\text { Ref. } \\
\text { photometry } \\
\text { (13) }\end{array}$ \\
\hline Coronet & $\ldots$ & $\ldots$ & $0.5-1$ & 15 & 15 & $3 / 13$ & $14 \pm 10$ & $2 / 8$ & $25 \pm 18$ & $1 / 2$ & $50 \pm \ldots$ & 15 \\
\hline$\rho \mathrm{Oph}$ & $0.5-3$ & 1 & $1-2$ & 16 & 35 & $2 / 123$ & $1.6 \pm 1.2$ & $2 / 90$ & $2.2 \pm 1.6$ & $0 / 24$ & $0 \pm \ldots$ & 47 \\
\hline NGC 2068/70 & $\cdots$ & $\cdots$ & $\sim 2$ & 17 & 17 & $7 / 51$ & $14 \pm 5$ & $7 / 40$ & $17 \pm 7$ & $5 / 7$ & $71 \pm 32$ & 17 \\
\hline NGC 2244 & $\sim 2$ & 2 & $\sim 2$ & 18 & 36 & $15 / 95$ & $16 \pm 4$ & $13 / 78$ & $17 \pm 5$ & $7 / 31$ & $23 \pm 9$ & 48 \\
\hline Serpens & $0.5-3$ & 3 & $1-3$ & 19 & 37 & $6 / 79$ & $7.6 \pm 3.1$ & $3 / 67$ & $4.5 \pm 2.6$ & $2 / 28$ & $7 \pm \ldots$ & 49 \\
\hline Taurus & $3-4$ & 4 & 1.5 & 20 & 38 & $10 / 152$ & $6.6 \pm 2.1$ & $8 / 129$ & $6.2 \pm 2.2$ & $6 / 54$ & $11 \pm 5$ & 38 \\
\hline NGC 1333 & $\lesssim 6$ & 5 & 1 & 20,21 & 39 & $2 / 44$ & $4.5 \pm 3.2$ & $1 / 31$ & $3.2 \pm \ldots$ & $1 / 9$ & $11 \pm \ldots$ & 47 \\
\hline Chameleon I & $\sim 6$ & 6 & 2.6 & 23 & 40 & $4 / 27$ & $15 \pm 8$ & $3 / 20$ & $15 \pm 9$ & $2 / 5$ & $40 \pm \ldots$ & 23 \\
\hline IC 348 & $\sim 6$ & 2 & 2.5 & 22 & 41 & $11 / 65$ & $17 \pm 5$ & $8 / 47$ & $17 \pm 6$ & $7 / 30$ & $23 \pm 9$ & 50 \\
\hline$\sigma$ Ori & $\sim 6$ & 2 & $\sim 3$ & 24 & 24 & $2 / 6$ & $33 \pm \ldots$ & $2 / 4$ & $50 \pm \ldots$ & $1 / 1$ & $100 \pm \ldots$ & 24 \\
\hline Totals & $\cdots$ & $\cdots$ & $\cdots$ & $\cdots$ & $\cdots$ & $62 / 653$ & $9.5 \pm 1.2$ & $49 / 514$ & $9.5 \pm 1.4$ & $32 / 191$ & $17 \pm 3$ & \\
\hline Dist. $<1$ kpc & $\cdots$ & $\cdots$ & $\cdots$ & $\cdots$ & $\cdots$ & $47 / 560$ & $8.4 \pm 1.3$ & $36 / 436$ & $8.3 \pm 1.4$ & $25 / 160$ & $16 \pm 3$ & $\cdots$ \\
\hline IR Members & $\cdots$ & $\cdots$ & $\cdots$ & $\cdots$ & $\cdots$ & $112 / 1473$ & $7.6 \pm 0.7$ & $83 / 1163$ & $7.1 \pm 0.8$ & $54 / 470$ & $11.5 \pm 1.6$ & \\
\hline IC 2395 & 9 & 7 & 6 & 25 & 7 & $8 / 26$ & $31 \pm 11$ & $5 / 19$ & $26 \pm 12$ & $2 / 2$ & $100 \pm \ldots$ & 7 \\
\hline Ori OB1b & $\sim 9$ & 10 & $4-6$ & 27 & 27 & $5 / 14$ & $36 \pm 16$ & $3 / 11$ & $27 \pm 16$ & $1 / 2$ & $50 \pm \ldots$ & 27 \\
\hline TW Hya & 10 & 8,9 & 8 & 26 & 42 & $6 / 10$ & $60 \pm 25$ & $6 / 10$ & $60 \pm 25$ & $3 / 3$ & $100 \pm 60$ & 51 \\
\hline Upper Sco & 11 & 11 & 5 & 28 & 43 & $51 / 103$ & $50 \pm 7$ & $42 / 90$ & $47 \pm 7$ & $26 / 45$ & $57 \pm 11$ & 52 \\
\hline$\epsilon$ Cha & $\sim 11$ & 12 & $\sim 6$ & 49 & 49 & $4 / 9$ & $44 \pm 22$ & $3 / 7$ & $43 \pm 25$ & $2 / 5$ & $40 \pm 28$ & 51 \\
\hline$\eta$ Cha & 11 & 8 & $\sim 6$ & 31 & 44 & $1 / 6$ & $17 \pm \ldots$ & $1 / 6$ & $17 \pm \ldots$ & $1 / 1$ & $100 \pm \ldots$ & 31 \\
\hline NGC 2362 & 12 & 2 & 5 & 32 & 45 & $8 / 18$ & $44 \pm 16$ & $8 / 14$ & $57 \pm 20$ & $2 / 3$ & $67 \pm 47$ & 42 \\
\hline$\gamma$ Vel & $\sim 12$ & 13 & $\sim 5$ & 33 & 33 & $7 / 16$ & $44 \pm 17$ & $3 / 7$ & $43 \pm 25$ & $1 / 1$ & $100 \pm \ldots$ & 33 \\
\hline Totals & $\cdots$ & $\cdots$ & $\cdots$ & $\cdots$ & $\cdots$ & $90 / 202$ & $45 \pm 5$ & $71 / 164$ & $43 \pm 5$ & $38 / 62$ & $61 \pm 10$ & \\
\hline Dist. $<1$ kpc & $\cdots$ & $\cdots$ & $\cdots$ & $\cdots$ & $\cdots$ & $81 / 178$ & $46 \pm 5$ & $62 / 144$ & $43 \pm 6$ & $35 / 58$ & $60 \pm 10$ & $\cdots$ \\
\hline Ori OB1a & $\sim 15$ & 14 & $7-10$ & 27 & 27 & $1 / 3$ & $33 \pm \ldots$ & $1 / 2$ & $50 \pm \ldots$ & $1 / 1$ & $100 \pm \ldots$ & 27 \\
\hline LCC/UCL & $16-17$ & 11 & 16 & 34 & 46 & $3 / 4$ & $75 \pm 43$ & $1 / 2$ & $50 \pm \ldots$ & $0 / 1$ & 0 & 51 \\
\hline Totals & $\cdots$ & $\cdots$ & $\cdots$ & $\ldots$ & $\cdots$ & $4 / 7$ & $57 \pm 29$ & $2 / 4$ & $50 \pm 35$ & $1 / 2$ & $50 \pm \ldots$ & $\ldots$ \\
\hline
\end{tabular}

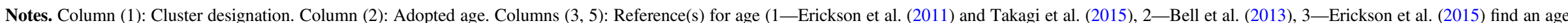

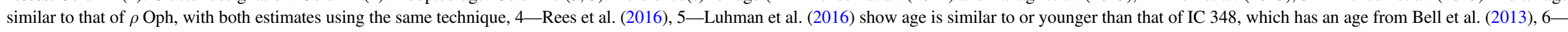

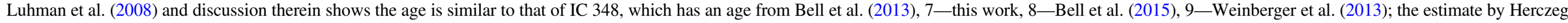

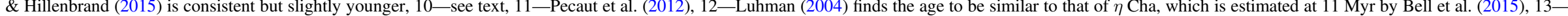

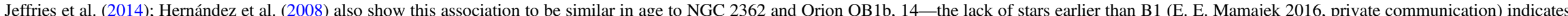

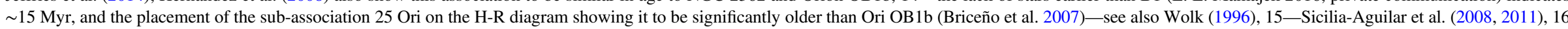

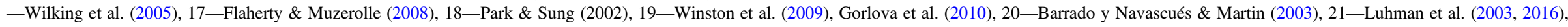

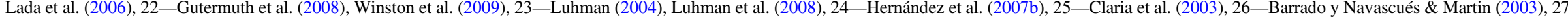

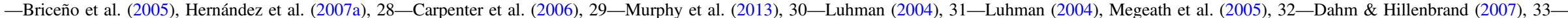

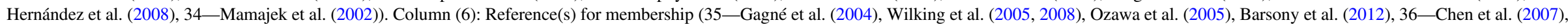

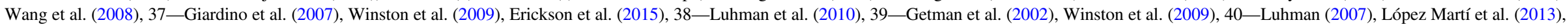

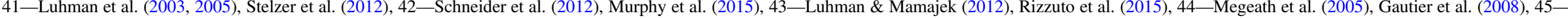

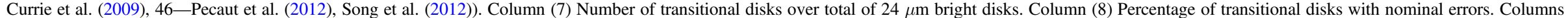

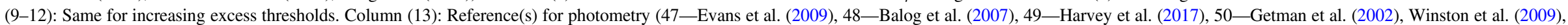
51-WISE, 52-Luhman \& Mamajek (2012) plus WISE). 
no compelling evidence for variations in the fraction of transitional disks over the full set of results reported in Table 6, although the case of $\rho$ Oph deserves further investigation.

Sicilia-Aguilar et al. (2008) have reported that the Coronet Cluster may have an abnormally high incidence of transitional disks, and indeed the counts reported for it in Table 6 are higher than average, albeit not at a statistically significant level. To understand any possible differences, we examine the seven transitional disks identified by Sicilia-Aguilar et al. (2008) in more detail. Only two of these disks are within the selection criteria for our sample-CrA-4111 and G-14. A third source on their list, G-65, has no data at $24 \mu \mathrm{m}$ (Table 3 of Sicilia-Aguilar et al. 2008) and furthermore has a [3.6] - [5.8] color of 1.01, far larger than our selection threshold of $<0.4$. Two more sources from their list, CrA-466 and G-87, also have large values of [3.6] - [5.8], 0.81 and 0.72 respectively. Two more from their list, CrA-205 and CrA-4109, are missing IRAC data used in our photometric selection; extrapolating from the existing data, they would likely be classified as transitional disks if full IRAC measurements were available. We do not add them to our sample because a similar detailed examination has not been performed for all the other clusters. However, we have included one source, G-30, which they excluded because of a low signalto-noise ratio $(\mathrm{S} / \mathrm{N})$ at $24 \mu \mathrm{m}$ (the indicated $\mathrm{S} / \mathrm{N}$ of 6.7 puts it above our WT threshold by $\sim 1 \sigma$; excluding it would introduce a potential bias in our method). If we added the two sources with missing IRAC data without subtracting the one with a low $\mathrm{S} / \mathrm{N}$, it would not raise the apparent excess of transitional disks to a statistically significant level. It is necessary to add three additional disks to the three we have identified to barely reach a $2 \sigma$ result. We conclude that the Coronet cluster does not provide a convincing counter-example to our conclusion that the incidence of transitional disks is not variable for stellar groupings of similar age, within the limits of the existing data.

\subsection{Change of Transitional Disk Proportion with Age}

The results of the preceding section suggest that we can use the average incidences of transitional disks for young, middleaged, and elderly systems without significant loss of information. There is a substantial and highly statistically significant increase in the fraction of transitional disks among those disks with strong $24 \mu \mathrm{m}$ excesses, going from the young to the middle-aged clusters/associations. The elderly clusters/associations have an incidence perhaps similar to that for the middle-aged ones, but the low numbers in the elderly grouping make any firm conclusions impossible. Figure 10 shows this trend graphically. The trend of an increasing incidence of transitional disks with age has been found previously, e.g., by Muzerolle et al. (2010), but with marginal statistical significance for the older clusters/associations, and by Currie \& Sicilia-Aguilar (2011), again with sparse representation of older clusters/associations, and with the results summarized in the review by Espaillat et al. (2014). This trend is put on a firm statistical basis by the average results for the young and middle-aged stellar clusters/assocations summarized in Table 6. In general, other studies using different criteria for identifying transitional disks will find them in different numbers (see cautions in Espaillat et al. 2014), but our study remains valid in terms of trends because it uses a homogeneous selection throughout.

What are the implications of the substantial change in transitional disks as a fraction of disks with strong infrared

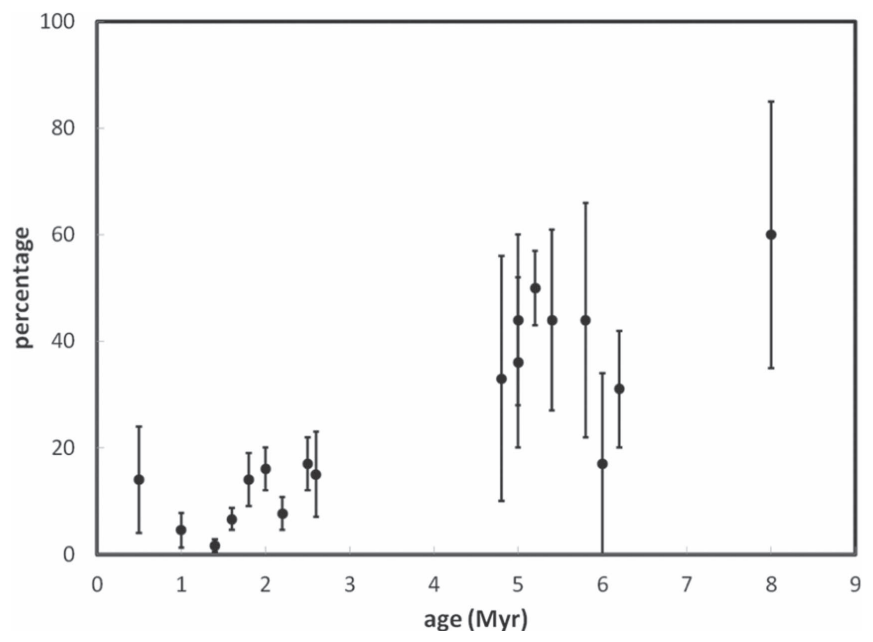

Figure 10. Percentage of transitional disks vs. age. defined as No. Transitional/(No. Transitional + No. Class II) $\times 100$. This graph uses the traditional age scale because it is more complete for the relevant clusters/ associations.

excess? There are two limiting possibilities: (1) that there is a systematic change in disk properties with age that is reflected by an increasing probability of any given disk entering this stage; or (2) that the disks retain similar structures but there is an increasing fraction of those remaining at any time that enters the transitional stage. An indication that the second case is closer to correct is that the relative fractions of disks with [8] $[24]>1.5,2.5$, or 3.5 are virtually identical for the averages for the young and middle-aged stellar groupings, i.e., $1: 0.98 \pm 0.21: 1.78 \pm 0.42$ and $1: 0.93 \pm 0.34: 1.35 \pm$ 0.28 , respectively (based on the boldfaced values in Table 6). Since the excess at $24 \mu \mathrm{m}$ is an indicator for the optical depth of the disks outside the inner few au, these statistics indicate that a minority of systems can retain disks that are still very dense in this zone for $10 \mathrm{Myr}$ or a bit longer.

We therefore assume that the time to clear an optically thick disk is independent of the age of the stellar grouping to which it belongs, and that such clearing passes through a transitional disk stage, which is of similar duration for all disks (e.g., Muzerolle et al. 2010; Espaillat et al. 2014). The consequence of the increase in transitional disk incidence is then that the decay of the $24 \mu \mathrm{m}$ dominant, optically thick disk component of a YSO population cannot be exponential, unlike that at shorter infrared wavelengths (e.g., Ribas et al. 2014), but must start slowly and accelerate relative to an exponential.

\section{CONCLUSIONS}

The open cluster IC 2395 can add significantly to our understanding of protoplanetary and early debris disk evolution, since it is relatively close $(800 \mathrm{pc})$ and at a critical age where protoplanetary disks are disappearing and debris disks begin to dominate. However, the cluster has largely been overlooked in disk studies. We report optical and infrared photometry and high-resolution optical spectroscopy of the cluster, from which we:

1. Increase the list of probable members to nearly 300, spanning spectral types from early B to middle M;

2. Estimate an age of $9 \pm 3 \mathrm{Myr}$ on the revised age scale, e.g., that of Bell et al. (2013); this value compares 
with $6 \pm 2 \mathrm{Myr}$ on the traditional scale (Claria et al. 2003); and

3. Identify 18 Class II candidates $(6.5 \%$ of the members with full IRAC data), eight transitional disk candidates $(2.9 \%)$, and 23 debris disk candidates $(8.3 \%)$.

We have combined the transitional disk information with homogeneously defined similar objects in 19 additional young clusters and associations to quantify the evolution of this phase, finding that

1. The dominant cause of variations in the proportion of transitional disks is age; most clusters of similar age have similar proportions of transitional disks among the systems with strong $24 \mu \mathrm{m}$ excesses. The single possible exception is $\rho$ Oph, where transtional disks are relatively rare.

2. The relative numbers of disks with different degrees of $24 \mu \mathrm{m}$ excess do not change significantly with age, implying that the change in the proportion of transitional disks is not driven by a systematic change of disk properties, e.g., a thinning of disks that makes them more susceptible to dissipation.

3. The number of disks in the transitional phase as a fraction of the total with strong $24 \mu \mathrm{m}$ excesses ([8] $-[24] \geqslant 1.5)$ increases from $(8.4 \pm 1.3) \%$ at $\sim 3 \mathrm{Myr}$ to $(46 \pm 5) \%$ at $\sim 10 \mathrm{Myr}$; alternative definitions of transitional disks will yield different percentages but should show the same trend.

4. Under the conventional assumption that the lifetime of the transitional stage is fixed, and given the evidence that the nature of the individual Class II and transitional disks does not change with age, this result implies that the decay in the proportion of systems with strong $24 \mu \mathrm{m}$ excesses cannot be exponential, but must start more slowly and finish more rapidly than the "best fit" exponential.

We have also demonstrated that IC 2395 is a rich cluster at a critical age for circumstellar disk evolution, worthy of additional study.

We thank Eric Mamajek for assistance on estimating cluster ages and Lynne Hillenbrand for a short course on the intricacies of current age determination. We also thank the anonymous referee for a detailed critique that yielded significant improvements in the paper. Partial support for this work was provided by NASA through Contract Number 1255094 issued by JPL/ Caltech. L.L.K. has been supported by the Lendület Young Researchers Program of the Hungarian Academy of Sciences. This research has made use of the SIMBAD database, operated at CDS, Strasbourg, France. This publication makes use of data products from the Two Micron All Sky Survey, which is a joint project of the University of Massachusetts and the Infrared Processing and Analysis Center/California Institute of Technology, funded by the National Aeronautics and Space Administration and the National Science Foundation. It also makes use of data products from the Wide-field Infrared Survey Explorer, which is a joint project of the University of California, Los Angeles, and the Jet Propulsion Laboratory/ California Institute of Technology, funded by the National Aeronautics and Space Administration. We thank the AngloAustralian Observatory and Cerro Tololo Inter-American
Observatory for granting telescope time and for logistical support of our program.

\section{REFERENCES}

Alexander, R. D., Clarke, C. J., \& Pringle, J. E. 2006, MNRAS, 369, 229

Allen, L. E., Calvet, N., D’Alessio, P., et al. 2004, ApJS, 154, 363

Allen, L. E., Megeath, S. T., Gutermuth, R., et al. 2007, in Planets and Protostars V, Vol. 951 ed. B. Reipurth, D. Jewit, \& K. Keil (Tucson, AZ: Univ. Arizona Press), 361

Balog, Z., Kiss, L. L., Vinkó, J., et al. 2009, ApJ, 698, 1989

Balog, Z., Muzerolle, J., Rieke, G. H., et al. 2007, ApJ, 660, 1532

Barrado y Navascués, D., \& Martin, E. L. 2003, AJ, 126, 2997

Barsony, M., Haisch, K. E., Marsh, K. A., \& McCarthy, C. 2012, ApJ, 751, 22 Baumgardt, H., Dettbarn, C., \& Wielen, R. 2000, A\&AS, 146, 251

Bell, C. P. M., Mamajek, E. E., \& Naylor, T. 2015, MNRAS, 454, 593

Bell, C. P. M., Naylor, T., Mayne, N. J., Jeffries, R. D., \& Littlefair, S. P. 2013, MNRAS, 434, 806

Bell, C. P. M., Rees, J. M., Naylor, T., et al. 2014, MNRAS, 445, 3496

Bevington, P. R., \& Robinson, D. K. 1992, Data Reduction and Error Analysis for the Physical Sciences (2nd ed.; New York: McGraw-Hill)

Briceño, C., Calvet, N., Hernández, J., et al. 2005, AJ, 129, 907

Briceño, C., Hartmann, L., Hernández, J., et al. 2007, ApJ, 661, 1119

Brown, A. G. A., Arenou, F., van Leeuwen, F., Lindegren, L., \& Luri, X. 1997, in ESA Special Publication No. 402, Hipparcos-Venice '97, ed. R. M. Bonnet et al., 63

Carpenter, J. M., Mamajek, E. E., Hillenbrand, L. A., \& Meyer, M. R. 2006, ApJL, 651, L49

Castelli, F., \& Kurucz, R. L. 2004, A\&A, 419, 725

Chen, L., de Grijs, R., \& Zhao, J. L. 2007, AJ, 134, 1368

Cieza, L. A., Schreiber, M. R., Romero, G. A., et al. 2012, ApJ, 750, 157

Claria, J. J., Lapasset, E., Piatti, A. E., \& Ahumada, A. V. 2003, A\&A, 409,541

Crawford, D. L., \& Mander, J. 1966, AJ, 71, 114

Currie, T., Lada, C. J., Plavchan, P., et al. 2009, ApJ, 698, 1

Currie, T., \& Sicilia-Aguilar, A. 2011, ApJ, 732, 24

Dahm, S. E., \& Hillenbrand, L. A. 2007, AJ, 133, 2072

Dias, W. S., Alessi, B. S., Moitinho, A., \& Lépine, J. R. D. 2002, A\&A, 389,871

Dias, W. S., Assafin, M., Flório, V., Alessi, B. S., \& Líbero, V. 2006, A\&A, 446, 949

Dias, W. S., Lépine, J. R. D., \& Alessi, B. S. 2001, A\&A, 376, 441

Dominik, C., \& Decin, G. 2003, ApJ, 598, 626

Ekström, S., Georgy, C., Eggenberger, P., et al. 2012, A\&A, 537A, 146

Engelbracht, C. W., Blaylock, M., Su, K. Y. L., et al. 2007, PASP, 119, 994

Erickson, K. L., Wilking, B. A., Meyer, M. R., et al. 2011, AJ, 142, 140

Erickson, K. L., Wilking, B. A., Meyer, M. R., et al. 2015, AJ, 149, 103

Espaillat, C., Muzerolle, J., Najita, J., et al. 2014, in Protostars and Planets VI, ed. H. Beuther et al. (Tucson, AZ: Univ. Arizona Press), 497

Evans, N. J., II, Dunham, M. M., Jorgensen, J. K., et al. 2009, ApJS, 181, 321

Fazio, G. G., Hora, J. L., Allen, L. E., et al. 2004, ApJS, 154, 10

Flaherty, K. M., \& Muzerolle, J. 2008, AJ, 135, 966

Flaherty, K. M., Pipher, J. L., Megeath, S. T., et al. 2007, ApJ, 663, 1069

Gagné, M., Skinner, S. L., \& Daniel, K. J. 2004, ApJ, 613, 393

Gáspár, A., Rieke, G. H., \& Balog, Z. 2013, ApJ, 768, 25

Gautier, T. N., III, Rebull, L. M., Stapelfeldt, K. R., \& Mainzer, A. 2008, ApJ, 683,813

Getman, K. V., Feigelson, E. D., Townsley, L., et al. 2002, ApJ, 575, 354

Giardino, G., Favata, F., Micela, G., Sciortino, S., \& Winston, E. 2007, A\&A, 463,275

Girardi, L., Grebel, E. K., Odenkirchen, M., \& Chiosi, C. 2004, A\&A, 422, 205

Gordon, K. D., Rieke, G. H., Engelbracht, C. W., et al. 2005, PASP, 117, 503

Gorlova, N., Balog, Z., Rieke, G. H., et al. 2007, ApJ, 670, 516

Gorlova, N., Steninhauer, A., \& Lada, E. 2010, ApJ, 716, 634

Goto, M., Usuda, T., Dullemond, C. P., et al. 2006, ApJ, 652, 758

Gott, J. R. I., Vogeley, M. S., Podariu, S., \& Ratra, B. 2001, ApJ, 549, 1

Gulyaev, A. P., \& Nesterov, V. V. (ed.) 1992, On a Catalogue of Four Million Stars (Moskow: Gosudarstvennyj Astronomicheskij Institut im.P.K. Shternberga, Izdatel'stvo Moskovskogo Universiteta), 72

Gutermuth, R. A., Megeath, S. T., Myers, P. C., et al. 2009, ApJS, 184, 18

Gutermuth, R. A., Myers, P. C., Megeath, S. T., et al. 2008, ApJ, 674, 336

Haisch, K. E., Lada, E. A., \& Lada, C. J. 2001, ApJ, 553, 153

Hartmann, L., Megeath, S. T., Allen, L. E., et al. 2005, ApJ, 629, 881

Harvey, P., Merín, B., Huard, T. L., et al. 2017, ApJ, 663, 1149

Herczeg, G. J., \& Hillenbrand, L. A. 2015, ApJ, 808, 23

Hernández, J., Calvet, N., Briceño, C., et al. 2007a, ApJ, 671, 1784 
Hernández, J., Hartmann, L., Calvet, N., et al. 2008, ApJ, 686, 1195 Hernández, J., Hartmann, L., Megeath, T., et al. 2007b, ApJ, 662, 1067 Hillenbrand, L. A. 2005, arXiv:astro-ph/0511083

Hogg, D. W., Neugebauer, G., Cohen, J. G., et al. 2000, AJ, 119, 1519

Hollenbach, D. J., Yorke, H. W., \& Johnstone, D. 2000, in Protostars and Planets IV, ed. V. Mannings, A. P. Boss, \& S. S. Russell (Tucson, AZ: Univ. Arizona Press), 401

Jeffries, R. D., Jackson, R. J., Cottaar, M., et al. 2014, A\&A, 563A, 94

Kennedy, G. M., \& Kenyon, S. J. 2014, ApJ, 695, 1210

Kenyon, S. J., \& Hartmann, L. 1995, ApJS, 101, 117

Kerschbaum, F., \& Hron, J. 1994, A\&A, 106, 397

Kharchenko, N. V. 2004, KFNT, 20, 366

Kharchenko, N. V., Pakulyak, L. K., \& Piskunov, A. E. 2003, ARep, 47, 263

Kharchenko, N. V., Piskunov, A. E., Röser, S., Schilbach, E., \& Scholz, R.-D. 2005, A\&A, 438, 1163

Kim, K. H., Watson, D. M., Manoj, P., et al. 2013, ApJ, 769, 149

Lada, C. J. 1987, in Proc. IAU Symp. 115, Star Forming Regions, ed. M. Peimbert \& J. Jugaku (Dordrecht: Reidel), 1

Lada, C. J., Muench, A. A., Luhman, K. L., et al. 2006, AJ, 131, 1574

Lada, C. J., \& Wilking, B. A. 1984, ApJ, 287, 610

Lagrange, A.-M., Backman, D. E., \& Artymowicz, P. 2000, in PPIV, ed. Mannings, Boss, \& Russell (Tucson, AZ: Univ. Arizona Press), 639

Landolt, Arlo. U. 1992, AJ, 104, 340

Lejeune, T., \& Schaerer, D. 2001, A\&A, 366, 538

Loktin, A. V., \& Beshenov, G. V. 2003, ARep, 47, 6

López Martí, B., Jiménez-Esteban, F., Bayo, A., et al. 2013, A\&A, 556A, 144

Luhman, K. L. 2004, ApJ, 602, 816

Luhman, K. L. 2007, ApJS, 173, 104

Luhman, K. L., Allen, L. E., Allen, P. R., et al. 2008, ApJ, 675, 1375

Luhman, K. L., Allen, P. R., Espaillat, C., Hartmann, L., \& Calvet, N. 2010, ApJS, 186, 111

Luhman, K. L., Esplin, T. L., \& Loutrel, N. P. 2016, arXiv:1605.08907

Luhman, K. L., Lada, E. A., Muench, A. A., \& Elston, R. J. 2005, ApJ, 618,810

Luhman, K. L., \& Mamajek, E. E. 2012, ApJ, 758, 31

Luhman, K. L., Stauffer, J. R., Muench, A. A., et al. 2003, ApJ, 593, 1093

Mamajek, E. E. 2005, ApJ, 634, 1385

Mamajek, E. E. 2009, in AIP Conf. Proc. 1158, Exoplanets and Disks: Their Formation and Diversity, ed. T. Usuda, M. Tamura, \& M. Ishii (Melville, NY: AIP), 3

Mamajek, E. E. 2015, A Modern Mean Dwarf Stellar Color and Effective Temperature Sequence (Rochester, NY: Univ. of Rochester) http://www. pas.rochester.edu/ emamajek/EEM_dwarf_UBVIJHK_colors_Teff.txt

Mamajek, E. E., Meyer, M. R., Hinz, P. M., et al. 2004, ApJ, 612, 496

Mamajek, E. E., Meyer, M. R., \& Liebert, J. 2002, AJ, 124, 1670

Manara, C. F., Fedele, D., Herczeg, G. J., \& Teixeira, P. S. 2015, A\&A, $585 \mathrm{~A}, 136$

Marigo, P., Girardi, L., Bressan, A., et al. 2008, A\&A, 482, 883

Megeath, S. T., Allen, L. E., Gutermuth, R. A., et al. 2004, ApJS, 154, 367

Megeath, S. T., Gutermuth, R., Muzerolle, J., et al. 2012, AJ, 144, 192

Megeath, S. T., Hartmann, L., Luhman, K., et al. 2005, ApJ, 634, 113

Meng, H. Y. A., Rieke, G. H., Su, K. Y. L., \& Gáspár, A. 2016, ApJ, submitted

Morris, S. L. 1985, ApJ, 295, 143

Murphy, S. J., Lawson, W. A., \& Bento, J. 2015, MNRAS, 453, 2220

Murphy, S. J., Lawson, W. A., \& Bessell, M. S. 2013, MNRAS, 435, 1325

Muzerolle, J., Allen, L. E., Megeath, S. T., Hernández, J., \& Guthermuth 2010 ApJ, 708, 1107

Muzerolle, J., Calvet, N., Hartmann, L., \& D’Alessio, P. 2003, ApJL, 597, L149

Olson, B. I. 1975, PASP, 87, 349

Ozawa, H., Grosso, N., \& Montmerle, T. 2005, A\&A, 438, 661

Palla, F., \& Stahler, S. W. 1999, ApJ, 525, 772

Papovich, C., Dole, H., Egami, E., et al. 2004, ApJS, 154, 70
Park, B.-G., \& Sung, H. 2001, AJ, 123, 892

Pascucci, I., Gorti, U., Hollenbach, D., et al. 2006, ApJ, 651, 1177

Paunzen, E. 2015, A\&A, 580A, 23

Pecaut, M. J., \& Mamajek, E. E. 2013, ApJS, 208, 9

Pecaut, M. J., Mamajek, E. E., \& Bubar, E. J. 2012, ApJ, 746, 154

Pickles, A., \& Depagne, É. 2010, PASP, 122, 1437

Platais, I., Kozhurina-Platais, V., Barnes, S., et al. 2001, AJ, 122, 1486

Quillen, A. C., Blackman, E. G., Frank, A., \& Varniere, P. 2004, ApJL, 612, L137

Reach, W. T., Megeath, S. T., Cohen, M., et al. 2005, PASP, 117, 978

Rees, J. M., Wilson, T., Bell, C. P. M., Jeffries, R. D., \& Naylor, T. 2016, in IAU Symp. 314, Young Stars \& Planets Near the Sun (Cambridge: Cambridge Univ. Press), 205

Ribas, Á., Merín, B., Bouy, H., \& Maud, L. 2014, A\&A, 561A, 54

Rice, W. K. M., Wood, K., Armitage, P. J., Whitney, B. A., \& Bjorkman, J. E. 2003, MNRAS, 342, 79

Rieke, G. H., Young, E. T., Engelbracht, C. W., et al. 2004, ApJS, 154, 25

Rizzuto, A. C., Ireland, M. J., \& Kraus, A. L. 2015, MNRAS, 448, 2737

Robitaille, T. P., Whitney, B. A., Indebetouw, R., Wood, K., \& Denzmore, P. 2006, ApJS, 167, 256

Schneider, A., Melis, C., \& Song, I. 2012, ApJ, 754, 39

Sicilia-Aguilar, A., Hartmann, L., Calvet, N., et al. 2006, ApJ, 638, 897

Sicilia-Aguilar, A., Hartmann, L. W., Hernández, J., Briceño, C., \& Calvet, N. 2005, AJ, 130, 188

Sicilia-Aguilar, A., Henning, T., Juhász, A., et al. 2008, ApJ, 687, 1145

Sicilia-Aguilar, A., Henning, T., Kainulainen, J., \& Roccatagliata, V. 2011, ApJ, 736, 137

Sierchio, J. M., Rieke, G. H., Su, K. Y. L., \& Gáspár, A. 2014, ApJ, 785, 33

Simon, M., \& Prato, L. 1995, ApJ, 450, 824

Skiff, B. A. 2014, yCat, 1, 2023

Skrutskie, M. F., Dutkevitch, D., Strom, S. E., et al. 1990, AJ, 99, 1187

Soderblom, D. R., Hillenbrand, L. A., Jeffres, R. D., Mamajek, E. E., \& Naylor, T. 2014, in Protostars and Planets VI, ed. Beuther et al. (Tucson, AZ: Univ. Arizona Press), 219

Song, I., Zuckerman, B., \& Bessell, M. S. 2012, AJ, 144, 8

Steinmetz, M., Zwitter, T., Siebert, A., et al. 2006, AJ, 132, 1645

Stelzer, B., Preibisch, T., Alexander, F., et al. 2012, A\&A, 537A, 135

Strom, K. M., Strom, S. E., Edwards, S., Cabrit, S., \& Skrutskie, M. F. 1989, AJ, 97, 1451

Takagi, Y., Itoh, Y., Arai, A., Sai, S., \& Oasa, Y. 2015, PASJ, 67, 87

Urban, L. E., Rieke, G. H., Su, K. Y. L., \& Trilling, D. E. 2012, ApJ, 750,98

Wang, J., Townsley, L. K., Feigelson, E. D., et al. 2008, ApJ, 675, 464

Wang, S. W., \& Looney, S. W. 2007, ApJ, 659, 1360

Weinberger, A. J., Anglada-Escudé, G., \& Boss, A. 2013, ApJ, 762, 118

Weinberger, A. J., Becklin, E. E., Song, I., \& Zuckermen, B. 2011, ApJ, 726, 72

Werner, M. W., Roellig, T. L., Low, F. J., et al. 2004, ApJS, 154, 1

Wilking, B. A., Gagné, M., \& Allen, L. E. 2008, in Handbook of Star Forming Regions, Vol. II, ed. B. Reipurth (San Francisco, CA: ASP), 351

Wilking, B. A., Meyer, M. r., Robinson, J. G., \& Greene, T. P. 2005, AJ, 130, 1733

Williams, J. P., \& Cieza, L. A. 2011, ARA\&A, 49, 67

Wilner, D. J., d'Alessio, P., Calvet, N., Claussenm, M. J., \& Hartmann, L. 2005, ApJL, 626, L109

Winston, E., Megeath, S. T., Wolk, S. J., et al. 2009, AJ, 137, 4777

Wolk, S. J. 1996, PhDT, State Univ. New York

Wolk, S. J., \& Walter, F. M. 1996, AJ, 111, 2066

Wyatt, M. C. 2008, ARA\&A, 46, 339

Yasui, C., Kobayashi, N., Tokunaga, A. T., \& Saito, M. 2014, MNRAS, 442, 2543

Zacharias, N., Monet, D. G., Levine, S. E., et al. 2004, BAAS, 205, 48.15

Zwitter, T., Siebert, A., Munari, U., et al. 2008, AJ, 136, 421 